OPEN ACCESS

Edited by:

Nick Swindale,

The University of British Columbia,

Canada

Reviewed by:

Yoshinao Kajikawa

Nathan Kline Institute for Psychiatric

Research, United States

Todd M. Mowery,

New York University, United States

*Correspondence:

Étienne de Villers-Sidani etienne.de-villers-sidani@mcgill.ca

Received: 09 July 2019 Accepted: 03 October 2019

Published: 23 October 2019

Citation:

Thomas ME, Guercio GD, Drudik KM and de Villers-Sidani $E$ (2019) Evidence of Hyperacusis in Adult Rats Following Non-traumatic

Sound Exposure.

Front. Syst. Neurosci. 13:55. doi: 10.3389/fnsys.2019.00055

\section{Evidence of Hyperacusis in Adult Rats Following Non-traumatic Sound Exposure}

\author{
Maryse E. Thomas ${ }^{1,2}$, Gerson D. Guercio ${ }^{3,4}$, Kristina M. Drudik ${ }^{1}$ and \\ Étienne de Villers-Sidani ${ }^{1,2 *}$
}

\begin{abstract}
${ }^{1}$ Montreal Neurological Institute, McGill University, Montreal, QC, Canada, ${ }^{2}$ Centre for Research on Brain, Language and Music, Montreal, QC, Canada, ${ }^{3}$ Department of Psychiatry, University of Minnesota Medical School, Minneapolis, MN, United States, ${ }^{4}$ Biomedical Sciences Institute, Federal University of Rio de Janeiro, Rio de Janiero, Brazil
\end{abstract}

Manipulations that enhance neuroplasticity may inadvertently create opportunities for maladaptation. We have previously used passive exposures to non-traumatic white noise to open windows of plasticity in the adult rat auditory cortex and induce frequencyspecific functional reorganizations of the tonotopic map. However, similar reorganizations in the central auditory pathway are thought to contribute to the generation of hearing disorders such as tinnitus and hyperacusis. Here, we investigate whether noise-induced reorganizations are accompanied by electrophysiological or behavioral evidence of tinnitus or hyperacusis in adult Long-Evans rats. We used a 2-week passive exposure to moderate-intensity (70 dB SPL) broadband white noise to reopen a critical period for spectral tuning such that a second 1-week exposure to $7 \mathrm{kHz}$ tone pips produced an expansion of the $7 \mathrm{kHz}$ frequency region in the primary auditory cortex (A1). We demonstrate for the first time that this expansion also takes place in the ventral auditory field (VAF). Sound exposure also led to spontaneous and sound-evoked hyperactivity in the anterior auditory field (AAF). Rats were assessed for behavioral evidence of tinnitus or hyperacusis using gap and tone prepulse inhibition of the acoustic startle response. We found that sound exposure did not affect gap-prepulse inhibition. However, sound exposure led to an improvement in prepulse inhibition when the prepulse was a $7 \mathrm{kHz}$ tone, showing that exposed rats had enhanced sensorimotor gating for the exposure frequency. Together, our electrophysiological and behavioral results provide evidence of hyperacusis but not tinnitus in sound-exposed animals. Our findings demonstrate that periods of prolonged noise exposure may open windows of plasticity that can also be understood as windows of vulnerability, potentially increasing the likelihood for maladaptive plasticity to take place.

Keywords: tonotopic map, maladaptive plasticity, tinnitus, hyperacusis, GPIAS, PPI, sound exposure, auditory cortex

\section{INTRODUCTION}

As recent decades of neuroscience research have revealed the brain's lifelong capacity for plastic change (Hofer et al., 2006; de Villers-Sidani and Merzenich, 2011), the goal of reopening critical periods (CPs) in order to stimulate learning and recovery in adulthood has become an important area of study. Researchers have already demonstrated the ability to reopen CPs in the auditory 
(Reed et al., 2011; Zhou et al., 2011; Blundon et al., 2017), visual (Pizzorusso et al., 2002; He et al., 2006; Harauzov et al., 2010), and somatosensory domains (Chung et al., 2017) in animal models. And steps have even been taken in humans, as the histonedeacetylase inhibitor, valproate, was found to reopen a $\mathrm{CP}$ for absolute pitch in adult non-musicians (Gervain et al., 2013). The inevitable quest for lifelong adaptability, however, should not be undertaken without considering the potential risks of opening windows of vulnerability on the brain.

One such vulnerability is the opportunity for maladaptive plasticity, which refers to structural or functional nervous system changes that disrupt normal function. Dysplastic symptoms such as hyperexcitation, altered neural connectivity, and topographic reorganizations can interfere with perceptual discrimination (O'Reilly et al., 2019), cause hypersensitivities or phantom percepts (Flor et al., 2001; Costigan et al., 2009; De Ridder et al., 2011), and contribute to chronic pain (Kuner and Flor, 2016). In the central auditory system, maladaptive plasticity is thought to underlie the generation of auditory disorders including chronic tinnitus and hyperacusis, the uncomfortable sensations of ringing in the ears and sound hypersensitivity. These potentially debilitating conditions usually emerge late in life comorbid with hearing loss and affect between $6 \%$ to $15 \%$ of the general population (Brozoski and Bauer, 2016). Although the exact neural underpinnings of tinnitus and hyperacusis remain elusive, their frequent co-occurrence with hearing loss points to the reduction of auditory inputs as a potential trigger for plasticity in spatially-defined regions of the auditory pathway (Eggermont and Roberts, 2004; Roberts et al., 2010; Langers et al., 2012). In animal models, tinnitus has primarily been associated with expanded representations of mid-to-high frequency regions, hypersynchronization, increased spontaneous firing, and increased burst firing in structures including the cochlear nucleus, inferior colliculus, and auditory cortex (Eggermont and Roberts, 2004; Roberts et al., 2010). Hyperacusis has been related to increased gain in the central auditory pathway in animal models detectable via higher spontaneous firing rates and sound-evoked potentials (Sun et al., 2012; Aazh et al., 2014; Hickox and Liberman, 2014). At present, some evidence links spontaneous and sound-evoked hyperactivity to tinnitus or hyperacusis in humans (Adjamian et al., 2009; $\mathrm{Gu}$ et al., 2010), but neuroimaging studies have yet to demonstrate macroscopic tonotopic reorganization in patients with tinnitus (Langers et al., 2012; Elgoyhen et al., 2015), illustrating that much remains to be understood in the etiology of both conditions.

Tinnitus has been tentatively linked to lifetime environmental noise exposure (Holgers and Pettersson, 2005; Guest et al., 2017; Moore et al., 2017). In adult rats and cats, prolonged moderateintensity sound exposures have been shown to produce strong experience-dependent plasticity altering tonotopic organization and auditory excitability (Pienkowski and Eggermont, 2009; Pienkowski et al., 2011; Zhou et al., 2011; Zheng, 2012). We have previously demonstrated that 2 weeks of passive exposure to moderate-intensity white noise can reopen windows of CP-like plasticity in the adult rat auditory cortex (Thomas et al., 2018).
We confirmed CP plasticity with a second passive exposure to pure tones that led to the expansion of the corresponding frequency region in the primary auditory cortex (A1). The perceptual consequences of this map expansion are incompletely understood and differ based on the mode of induction, with primarily sound-driven-as opposed to neuromodulatorydriven-expansions impairing discrimination for the exposure frequency (Han et al., 2007; Eggermont, 2013; Froemke et al., 2013). Based on the common phenotype of map expansion in both sound-exposed animals and animals with tinnitus, we wondered if the sound exposure used in our previous study could have imparted our rats with tinnitus or another auditory disorder.

In the present study, we investigated the possibility that cortical map expansion could be indicative of maladaptive plasticity in sound-exposed animals. To this end, we induced $7 \mathrm{kHz}$ map expansion in female adult Long Evans rats using continuous exposure to moderate-intensity (70 dB SPL) broadband white noise for 2 weeks followed by $7 \mathrm{kHz}$ tone pips for 1 week. We hypothesized that this exposure would lead to specific maladaptive plasticity in cortical regions that preferentially respond to $7 \mathrm{kHz}$ accompanied by behavioral evidence of hyperacusis or tinnitus as measured by prepulse inhibition (PPI) and gap-prepulse inhibition (GPIAS) of the acoustic startle reflex, respectively. We documented the effects of exposure on electrophysiological response properties in the A1, anterior auditory field (AAF), and ventral auditory field (VAF). We found evidence of hyperactivity in the AAF of exposed animals consistent with hyperacusis, which was supported by an improvement in PPI when the prepulse was a $7 \mathrm{kHz}$ pure tone. We did not find electrophysiological or behavioral evidence of tinnitus. Our findings indicate that although non-traumatic white noise exposure can open windows of plasticity on the brain, these can also be understood as windows of vulnerability that may increase the likelihood for maladaptive plasticity to occur.

\section{MATERIALS AND METHODS}

The experimental procedures used in this study were approved by the Montreal Neurological Institute Animal Care Committee and follow the guidelines of the Canadian Council on Animal Care.

\section{Sound Exposure}

Female 3- to 4-month-old Long-Evans rats were housed in sound-attenuated chambers under a $12 \mathrm{~h}$ light/dark cycle and given ad libitum access to food and water. Rats were assigned to either the naive or sound exposure condition. Naive rats $(N=23)$ had no acoustic manipulation of their environment (background sound level $40 \mathrm{~dB}$ SPL). Exposed rats $(N=25)$ were passively exposed to $70 \mathrm{~dB}$ SPL (decibels sound pressure level, RMS) continuous white noise for 2 weeks immediately followed by a 1-week exposure to trains of $7 \mathrm{kHz}$ tone pips. From each group, 12 rats were used for behavioral testing (12 Naïve$\mathrm{BEH}$ and 12 Exposed-BEH) while the remaining rats (11 Naive and 13 Exposed) were used for electrophysiological recordings. To reduce the number of animals sacrificed for this study, the electrophysiological data for the Exposed group came from 
combining two groups of noise $+7 \mathrm{kHz}$-exposed animals that underwent slightly different $7 \mathrm{kHz}$ exposures. Four rats came from Thomas et al. (2018) and were exposed to $7 \mathrm{kHz}$ pure tones. The other nine rats were exposed to $7 \mathrm{kHz}$ tone pip clouds consisting of pure tones of random frequencies within a $\frac{1}{4}$ octave range centered on $7.6 \mathrm{kHz}$ (ranging between 7 and $8.3 \mathrm{kHz}$ ). Other than tone frequencies, all other properties of the tone exposures were the same. The noise and tone pips were generated using custom MATLAB scripts (The MathWorks, Inc., Natick, MA, USA) and played through an Ultralite-mk3 Hybrid Interface (MOTU Inc., Cambridge, MA, USA) with sampling at $192 \mathrm{kHz}$. The noise stimuli were amplified to a free-field sound level calibrated so that the average stimulus intensity measured in the center of the chamber was $70 \mathrm{~dB}$ SPL. Tones were $50 \mathrm{~ms}$ in duration (5 ms onset and offset ramps) and delivered in trains of five pips per second. The interval between each train of tones was a random duration generated from a normal distribution with a mean of $2.5 \mathrm{~s}$. The tone pips were amplified to an intensity of $65 \mathrm{~dB}$ SPL measured in the center of the chamber. All stimuli were played $24 \mathrm{~h}$ per day for the duration of the exposure periods.

\section{Electrophysiological Recordings}

Electrophysiological recordings of the left auditory cortex were performed under isoflurane anesthesia in a shielded soundproof recording chamber. Rats were pre-medicated with dexamethasone $(0.2 \mathrm{mg} / \mathrm{kg}$, i.m.) to minimize brain edema. Anesthesia was induced with ketamine/xylazine/acepromazine $(63 / 13 / 1.5 \mathrm{mg} / \mathrm{kg}$, i.p.) followed by continuous delivery of isoflurane $1 \%$ in oxygen via endotracheal intubation and mechanical ventilation. Heart rate and blood oxygen saturation were monitored with a pulse oximeter. Body temperature was monitored with a rectal probe and maintained at $37^{\circ} \mathrm{C}$ with a homeothermic blanket system. Rats were held by the orbits in a custom-designed head holder leaving the ears unobstructed. The cisterna magna was drained of cerebrospinal fluid to further minimize cerebral edema. To access the auditory cortex, the left temporalis muscle was reflected, the skull over the auditory cortex was removed, and the dura was resected. Once exposed, the cortex was maintained under a thin layer of silicone oil to prevent desiccation. Acoustic stimuli were delivered in a free field manner to the right ear through a calibrated speaker. Cortical responses were recorded with a high-impedance 64-channel tungsten microelectrode array (Tucker-Davis Technologies, TDT, Alachua, FL, USA) lowered to a depth of $600-900 \mu \mathrm{m}$ (layers 4/5). The electrode wires (33 $\mu \mathrm{m}$ diameter) were arranged in an $8 \times 8$ grid orthogonal to the cortex spaced $375 \mu \mathrm{m}$ apart with row separation of $500 \mu \mathrm{m}$. To maximize recording density, neural responses were consecutively recorded from multiple positions within each rat. The stereotaxic location of each position relative to the first was noted in order to accurately reconstruct auditory maps during offline analysis. Extracellular multi-unit responses were obtained, amplified, and filtered $(0.3-5 \mathrm{kHz})$ using a TDT RZ2 processor. The TDT OpenEx software package was used to generate acoustic stimuli, monitor cortical activity online, and store data for offline analysis.

\section{Acoustic Stimulation}

Frequency-intensity receptive fields were constructed using neuronal responses to a range of frequency-intensity combinations of pure tones. Sixty-six frequencies $(0.75-70 \mathrm{kHz}$; 0.1 octave increments; $25 \mathrm{~ms}$ duration; $5 \mathrm{~ms}$ ramps) were presented at eight sound intensities $(0-70 \mathrm{~dB}$ SPL; $10 \mathrm{~dB}$ increments) at a rate of one tone per second with three repetitions and in random presentation order. The characteristic frequency $(\mathrm{CF})$ and threshold of a cortical site were defined, respectively, as the frequency and intensity at the tip of the $\mathrm{V}$-shaped tuning curve derived from peri-stimulus time histograms (PSTHs). For flat-peaked tuning curves or tuning curves with multiple peaks, the CF was defined as the frequency with the lowest threshold and the strongest firing rate. Response bandwidths $20 \mathrm{~dB}$ above the threshold of tuning curves (BW20) were measured for all sites. The onset latency, defined as the time in ms when the PSTH first exceeded mean baseline firing rate by 2.5 standard deviations, was also measured for each cortical site. The CF, threshold, BW20, and latencies were first determined by an automated custom MATLAB routine and then manually verified by an experimenter blind to the identity of the experimental groups. Cortical sites were identified as belonging to A1, AAF, VAF, or posterior auditory field (PAF) based on published functional characteristics of each field (Polley et al., 2007). These were reversal of tonotopic gradients, onset latencies, threshold, and PSTH morphologies (Supplementary Figure S1). To generate tonotopic maps, Voronoi tessellation was performed using custom MATLAB scripts to create tessellated polygons with electrode penetration sites at their centers.

\section{Neural Synchrony}

The degree of neural synchronization in the auditory cortex was computed from recordings of spontaneous neural activity that were at least 5 min long. Recordings with apparent burst suppression were not included in analyses. Burst suppression was characterized by periods of high spontaneous firing alternating with periods of no activity determined through visual inspection of the raster plots and continuous average firing rate. If a portion of any recording was deemed to have burst suppression, the recording was rejected. The average coefficient of variation $(\mathrm{CV})$ of the inter-spike interval-a measure of burstiness-corresponded well with our classification of burst suppression, as the mean CV was significantly higher for recordings identified as having burst suppression (mean $=3.45, \mathrm{SD}=0.15)$ than those that were not $[$ mean $=1.86$, $\mathrm{SD}=0.07$; mixed-effects one-way analysis of variance (ANOVA) $F_{(1,48.13)}=93.34, p<0.0001, n=2,070$ units within 53 positions and 24 rats]. Offline spike sorting was performed using TDT OpenSorter software to isolate single unit activity based on an automated Bayesian sorting algorithm. The success of the spike sorting algorithm was assessed by inspecting the number of refractory period violations for all identified clusters (Supplementary Figure S2). The fraction of spikes that fell within a $2 \mathrm{~ms}$ refractory period was calculated and it was found that $36.1 \%$ of all clusters had zero refractory period violations and $96.9 \%$ of all clusters had two or fewer violations per 100 spikes (Supplementary Figure S2A). An average of 1.63 units was 
identified per electrode channel. Example histograms of the interspike interval and the autocorrelation of spike times for representative units are presented in Supplementary Figure S2B, displaying a dearth of spikes occurring within the refractory period. In addition, the percentage of refractory period violations did not differ between experimental groups (Supplementary Figure S2C). These results indicate that there are a relatively small number of false-positive classifications present in the data, which are unlikely to affect experimental outcomes. Measures of synchronization were computed from binary spike events detected from A1 units in separate channels. Cross-correlograms were computed by counting the number of spike coincidences for pairs of spike trains for time lags of -500 to $500 \mathrm{~ms}$ with $1 \mathrm{~ms}$ bin size and normalized by dividing each bin by the square root of the product of the number of total discharges in each spike train (Eggermont, 1992).

\section{Behavioral Testing}

Behavioral testing took place during the day at the Glen Site of the McGill University Health Centre. At the end of the exposure period, each Naïve-BEH animal was randomly assigned to a pair with one Exposed-BEH animal. Once paired, the rats were transported in their original cages to a loading area by cart. There, they were transferred to a vehicle and driven to the Glen Site, approximately $25 \mathrm{~min}$ away. The rats were again transported to a holding area adjoining the behavioral testing facility by cart where they were acclimatized for a minimum of $2 \mathrm{~h}$. Rats remained covered for all of the steps above until they reached the holding area. The paired rats then underwent behavioral testing simultaneously in order of pairing (two rats were tested at a time). This procedure took place twice, with six animals from each group tested on each day. All behavioral data were collected in sound-attenuating chambers. Sounds were delivered from a free-field speaker and rats were free to roam the chamber. The acoustic startle response was measured using the LE 118-8 Startle and Fear Interface (Panlab, Barcelona, Spain). The startle pulse was a white noise burst (120 dB SPL, $40 \mathrm{~ms}$ ) for both GPIAS and PPI. For GPIAS, rats were acclimatized for $3 \mathrm{~min}$ in a pure tone background that was either $3.5 \mathrm{kHz}$ or $7 \mathrm{kHz}$ (65 dB SPL), followed by four randomly interleaved no-gap and gap (30 ms) trials (intertrial interval 12-30 s). During the gap trials, the gap preceded the pulse by $60 \mathrm{~ms}$. This procedure was performed three times, and startle activity for the no-gap and gap trials were averaged across a total of 12 trials each. For PPI, rats were acclimatized for $3 \mathrm{~min}$ in a white noise background (65 dB SPL). The subsequent experimental protocol consisted of 10 trials each of no stimulus, the startle pulse alone, and two prepulse frequencies (3.5 or $7 \mathrm{kHz}, 20 \mathrm{~ms}, 75 \mathrm{~dB} \mathrm{SPL})$ presented $60 \mathrm{~ms}$ before the startle pulse, in pseudorandom order (intertrial interval 12-30 s). The startle activity for the no stimulus, startle pulse, $3.5 \mathrm{kHz}$ prepulse, and $7 \mathrm{kHz}$ prepulse trials were averaged across the 10 trials. We calculated prepulse inhibition of the startle response using the formula: \%PPI = $100-$ (startle response for prepulse trials/startle response for startle pulse alone trials) $\times 100$. We calculated gap-prepulse inhibition of the startle response with the formula:
$\%$ GPIAS $=100-($ startle response for gap trials/startle response for no-gap trials) $\times 100$.

\section{Statistical Analyses}

For all statistical analyses, results are reported in parentheses including test name, statistic, and number of data points per level of nested data. Linear mixed-effects models (Reed and Kaas, 2010; Aarts et al., 2014) were used to analyze data collected through nested experimental designs (e.g., for synchronization analyses: neuron pair nested within recording position nested within rat). For these models, recording position nested within rat ID were included as random effects. A matched pairs design using paired $t$-tests was employed to analyze behavioral data in order to control for potential confounding effects of transport, handling, waiting, and testing times on the acoustic startle response (Geyer and Swerdlow, 1998; Longenecker and Galazyuk, 2012). Accordingly, the effect size calculated by Cohen's dav is reported for behavioral results (Lakens, 2013). Analyses were conducted using MATLAB and JMP 13 (SAS Institute, Cary, NC, USA). The mixed-effect test results are reported with the degrees of freedom denominator approximated for normal data using the Kenward-Roger adjustment. Unless otherwise stated, Tukey's test evaluated at an alpha level of 0.05 was used for all post hoc comparisons. Where applicable, back-transformed means derived from statistical models were plotted in figures. Where results are not shown in figures, means \pm standard error are reported in the text.

\section{RESULTS}

\section{Electrophysiological Correlates of Sound Exposure}

We documented the effects of 2 weeks of passive exposure to white noise followed by 1 week of $7 \mathrm{kHz}$ tone pip exposure on electrophysiological response properties in 13 rats (Exposed Group) and compared them to 11 rats that were housed in a standard acoustic environment (Naïve Group; Figure 1A). CF tonotopic maps were reconstructed from the left auditory cortex under isoflurane anesthesia using in vivo multiunit responses to presentations of tone pips of various frequencies and intensities (Figure 1B). Responsive sites were classified as belonging to A1, AAF, VAF, or PAF based on the published functional characteristics of each field (Polley et al., 2007; Profant et al., 2013), specifically reversal of tonotopic gradients, onset latencies, threshold, and PSTH morphologies (Supplementary Figure S1). Using functional properties alone, we were not able to distinguish VAF from the fifth rat auditory field, suprarhinal auditory field (SRAF), so any presumed VAF or SRAF site was classified under the common label of VAF. In addition, we did not conduct analyses on the data we obtained from PAF due to the difficulty of assigning a CF to most PAF units, which have broad and noisy tuning curves. For each animal, we determined whether we obtained full or partial A1, AAF, and VAF maps. A full map was defined by having low, medium, and high frequency regions as well as a reversal of the tonotopic gradient on one border and non-auditory sites on the opposite border. In Figure 1B, the representative CF maps from each group were selected for having 
A

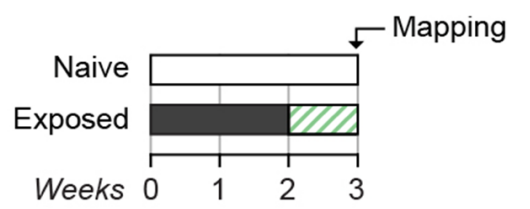

$\mathbf{B}$<smiles></smiles>

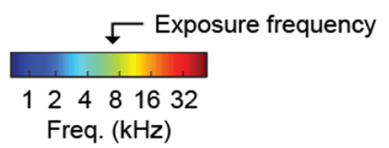

Freq. (kHz)

E
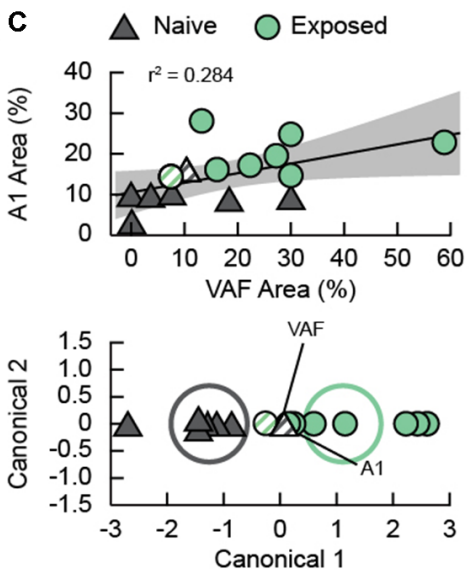

$\mathbf{D}$
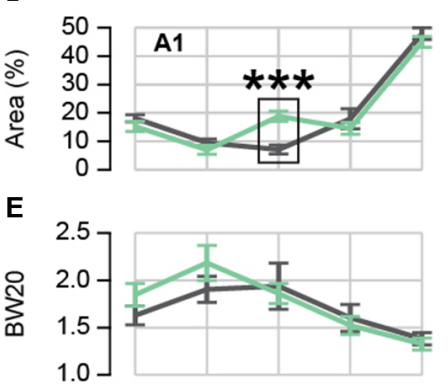

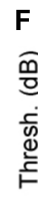

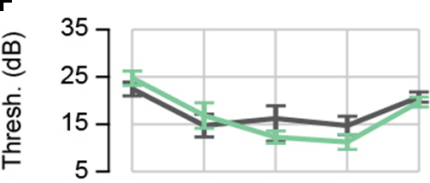

$\mathbf{G}$

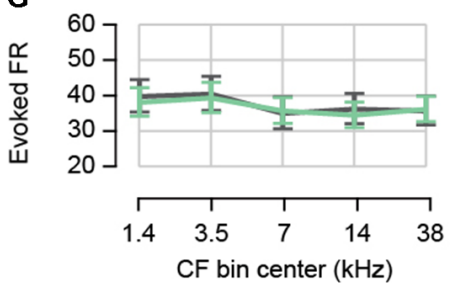

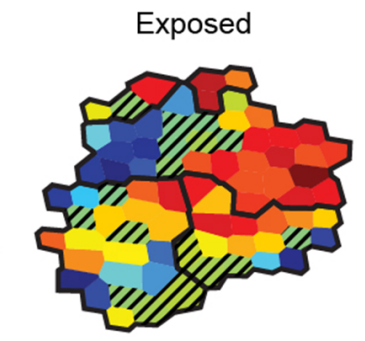
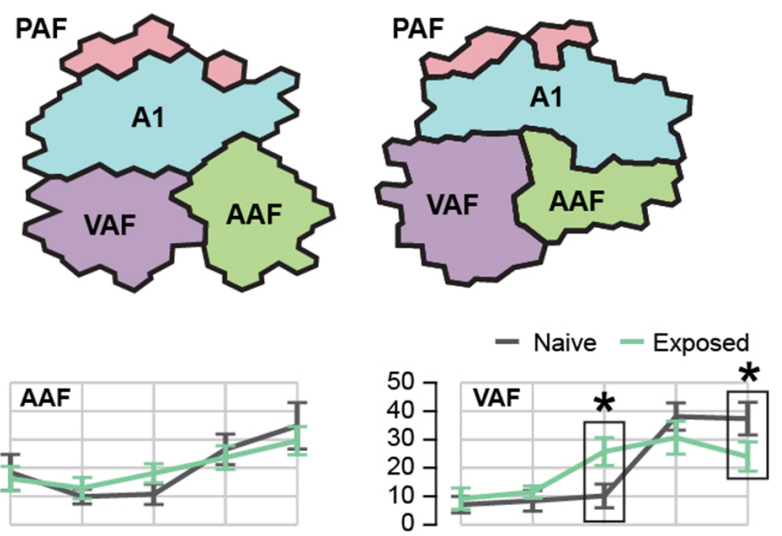

$7 \mathrm{kHz}$
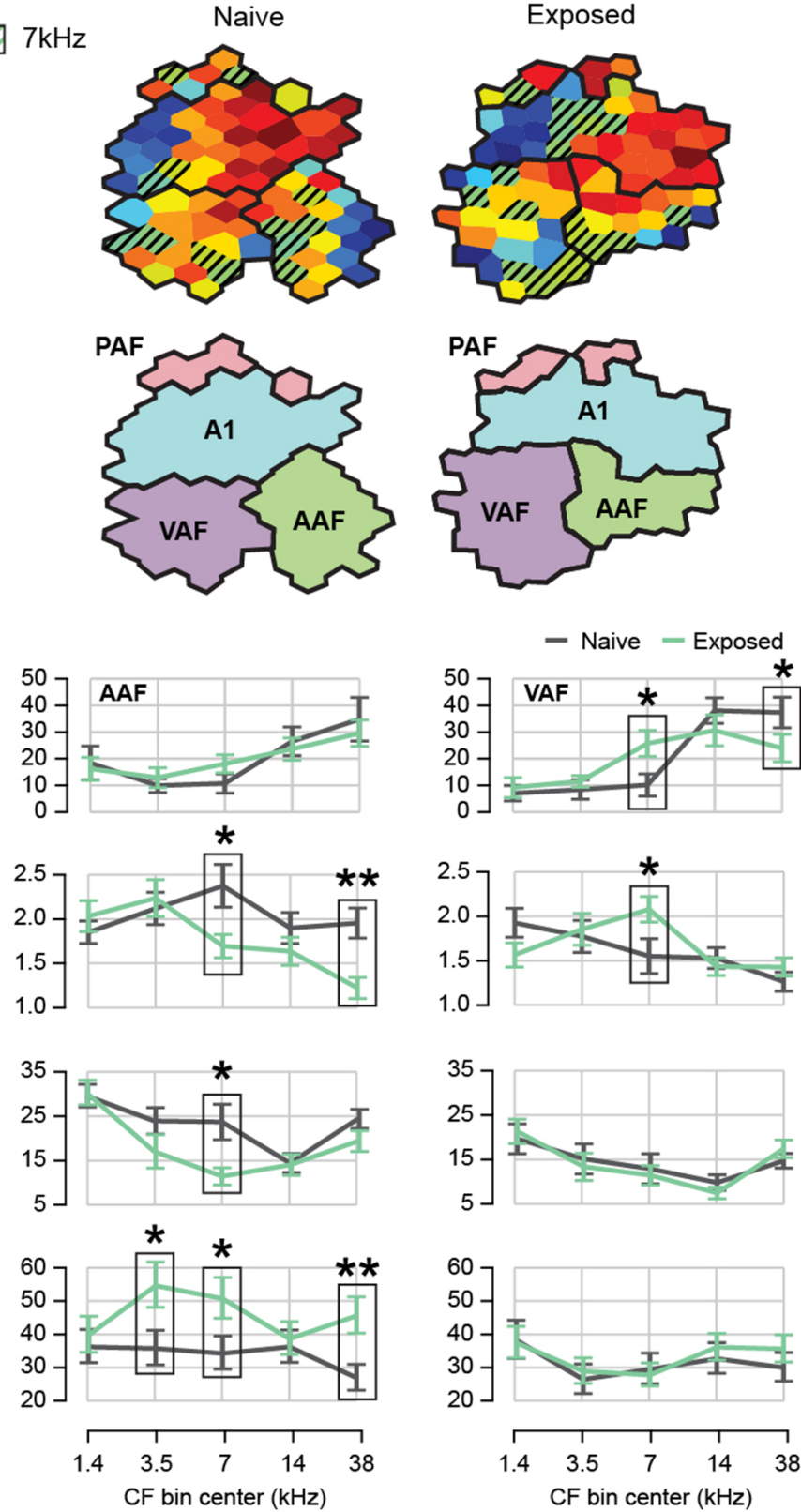
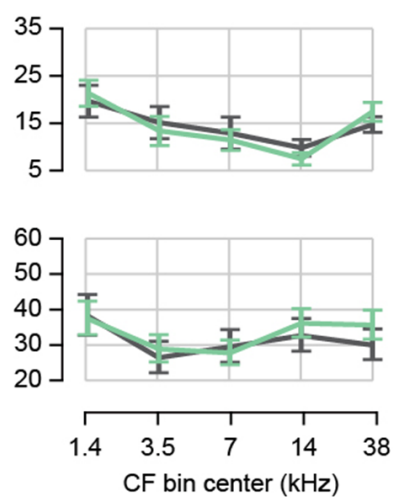

FIGURE 1 | Effect of sound exposure on cortical tuning and tone-evoked activity. (A) Sound exposure protocol. Naïve rats were housed in a normal acoustic environment while exposed rats were passively exposed to 2 weeks of moderate-intensity broadband white noise followed by 1 week of $7 \mathrm{kHz}$ tone pips. (B) An example characteristic frequency (CF) map from each experimental group containing all auditory fields. Hatched sites represent those with a $\mathrm{CF}$ of $7 \mathrm{kHz} \pm \frac{1}{2}$ octave. (C) Top: correlation between the percent A1 area and percent VAF area with a CF of $7 \mathrm{kHz} \pm \frac{1}{2}$ octave. Shaded region represents $95 \%$ confidence of fit for the regression. Bottom: canonical plot of the linear discriminant analysis based on the percent of A1 and VAF area with a CF of $7 \mathrm{kHz} \pm \frac{1}{2}$ octave. Rats were automatically classified as either naïve or exposed; hatched points identify rats that were misclassified. Ellipses represent the $95 \%$ confidence region for the true mean of each group. (D) Average map area with CF in five frequency bins. Only full auditory fields were used for map percentages. (E) Average BW20 for receptive fields with CF in five frequency bins. (F) Average cortical threshold for receptive fields with CF in five frequency bins. (G) Average tone-evoked firing rate for units with CF in five frequency bins. ${ }^{*} p<0.05,{ }^{* *} p<0.01,{ }^{* * *} p<0.001$. Error bars represent standard error of the mean (SEM). A1, primary auditory field; AAF, anterior auditory field; VAF, ventral auditory field; PAF, posterior auditory field. See Table $\mathbf{1}$ for number of rats, recording positions, and cortical sites per auditory field and group. 
TABLE 1 | Summary of data.

\begin{tabular}{|c|c|c|c|c|c|c|c|c|c|c|}
\hline & \multirow[b]{2}{*}{ Group } & \multirow[b]{2}{*}{ Field } & \multirow[b]{2}{*}{ Rats } & \multirow[b]{2}{*}{ Positions } & \multicolumn{6}{|c|}{ Sites/Units per CF bin } \\
\hline & & & & & 1.4 & 3.5 & 7 & 14 & 38 & Total \\
\hline \multirow[t]{10}{*}{ All data } & Naive & $\mathrm{A} 1$ & 10 & 22 & 55 & 29 & 21 & 52 & 155 & 312 \\
\hline & & AAF & 11 & 22 & 31 & 16 & 19 & 35 & 50 & 151 \\
\hline & & VAF & 10 & 18 & 15 & 15 & 17 & 48 & 52 & 147 \\
\hline & & PAF & 10 & 17 & - & - & - & - & - & 69 \\
\hline & & All & 11 & 24 & & & & & & 679 \\
\hline & Exposed & A1 & 13 & 30 & 47 & 29 & 67 & 59 & 187 & 389 \\
\hline & & AAF & 11 & 23 & 23 & 17 & 27 & 31 & 43 & 141 \\
\hline & & VAF & 13 & 27 & 19 & 21 & 46 & 62 & 47 & 195 \\
\hline & & PAF & 11 & 21 & - & - & - & - & - & 105 \\
\hline & & All & 13 & 30 & & & & & & 830 \\
\hline \multirow[t]{10}{*}{ Full fields only } & Naïve & $\mathrm{A} 1$ & 10 & 21 & 53 & 28 & 21 & 52 & 136 & 290 \\
\hline & & AAF & 7 & 16 & 22 & 15 & 18 & 32 & 44 & 131 \\
\hline & & VAF & 7 & 12 & 11 & 13 & 12 & 43 & 42 & 121 \\
\hline & & PAF & 5 & 10 & - & - & - & - & - & 49 \\
\hline & & All & 11 & 24 & & & & & & 591 \\
\hline & Exposed & $\mathrm{A} 1$ & 9 & 19 & 47 & 21 & 59 & 47 & 141 & 315 \\
\hline & & AAF & 8 & 19 & 23 & 17 & 25 & 27 & 37 & 129 \\
\hline & & VAF & 9 & 19 & 17 & 20 & 41 & 49 & 39 & 166 \\
\hline & & PAF & 8 & 18 & - & - & - & - & - & 99 \\
\hline & & All & 13 & 30 & & & & & & 709 \\
\hline \multirow[t]{10}{*}{ Sorted units } & Naïve & $\mathrm{A} 1$ & 10 & 19 & 77 & 32 & 34 & 77 & 197 & 417 \\
\hline & & AAF & 11 & 19 & 33 & 23 & 18 & 29 & 65 & 168 \\
\hline & & VAF & 9 & 15 & 17 & 17 & 17 & 62 & 76 & 189 \\
\hline & & PAF & 10 & 17 & - & - & - & - & - & 110 \\
\hline & & All & 11 & 20 & & & & & & 884 \\
\hline & Exposed & $\mathrm{A} 1$ & 13 & 25 & 76 & 36 & 90 & 80 & 263 & 545 \\
\hline & & AAF & 11 & 18 & 28 & 17 & 28 & 36 & 45 & 154 \\
\hline & & VAF & 13 & 22 & 25 & 33 & 62 & 91 & 59 & 270 \\
\hline & & PAF & 10 & 18 & - & - & - & - & - & 164 \\
\hline & & All & 13 & 25 & & & & & & 1,133 \\
\hline
\end{tabular}

Number of rats and recording positions from which data were obtained for each auditory field and experimental group. Number of cortical sites (or units, for sorted data) per CF bin in each field. PAF units were not assigned CFs.

full maps of each field. Table 1 lists the number of cortical sites obtained for each auditory field and experimental group for both full and partial maps.

We first compared the degree of $7 \mathrm{kHz}$ map expansion between exposed and naïve animals (Figures 1B-D). Using full field maps only, we calculated the percentage of map area with CFs in five frequency bins with centers at approximately 1.4, 3.5, 7,14 , and $38 \mathrm{kHz}$. The range of each bin was 1 octave except for the first and last bin, which were 1.7 and 1.8 octaves respectively. The bins were defined in relation to $7 \mathrm{kHz}$ in order to maximize specificity for the middle bins while covering the full range of recorded CFs. In A1, as expected, we observed a significantly greater percentage of map area tuned to $7 \mathrm{kHz}$ for the Exposed group (two-way ANOVA with Group and Bin as factors. Interaction $F_{(4,4)}=5.30, p=0.0007$ followed by simple main effects for $7 \mathrm{kHz} F_{(1,85)}=16.94, p<0.0001, n=19$ rats). This over-representation was not compensated by a consistent underrepresentation in another frequency bin as no other simple main effect was significant $\left(F_{(1,85)} \leq 1.41\right.$, all $\left.p \geq 0.2387\right)$. In AAF, we detected no difference in map area for any frequency bin (two-way ANOVA with Group and Bin as factors. Interaction: $F_{(4,4)}=0.55, p=0.6965, n=15$ rats), whereas in VAF we observed a significant over-representation of the $7 \mathrm{kHz}$ frequency bin for exposed animals, as well as a significant decrease in map area in the highest frequency bin (two-way ANOVA with Group and Bin as factors. Interaction $F_{(4,4)}=2.96, p=0.0257$ followed by simple main effects for $7 \mathrm{kHz} F_{(1,70)}=5.85, p=0.0182$ and $38 \mathrm{kHz} F_{(1,70)}=4.30, p=0.0418, n=16$ rats). No other frequency bin was significantly changed (simple main effects $F_{(1,70)} \leq$ 1.33 , all $p \geq 0.2521$ ). To ensure that we did not oversample the $7 \mathrm{kHz}$ frequency region in the Exposed group, we compared the average distance between each site and its nearest neighbor from full field maps. We observed no significant differences in nearest-neighbor distance between Naïve and Exposed animals in any frequency bin for any field, confirming that differences in frequency representation were not due to differences in sampling (mixed-effects two-way ANOVAs with Group and Bin as factors. A1: mean distance Naïve $=323.72 \pm 6.82 \mu \mathrm{m}$, Exposed $=326.21 \pm 7.14 \mu \mathrm{m}$, Interaction $F_{(4,579)}=1.00, p=4060$. Main effect of Group $F_{(1,17.26)}=0.06, p=0.8038, n=605$ sites 
within 19 rats. AAF: mean distance Naïve $=336.20 \pm 11.56 \mu \mathrm{m}$, Exposed $=329.73 \pm 10.77 \mu \mathrm{m}$, Interaction $F_{(4,246.8)}=1.04$, $p=0.3863$. Main effect of Group $F_{(1,13.31)}=0.17$, $p=0.6887, n=260$ sites within 15 rats. VAF: mean distance Naïve $=355.58 \pm 11.85 \mu \mathrm{m}$, Exposed $=338.55 \pm 10.09 \mu \mathrm{m}$, Interaction $F_{(4,269.5)}=1.73, p=0.1445$. Main effect of Group $F_{(1,14.9)}=1.20, p=0.2911, n=287$ sites within 16 rats $)$. The above results document for the first time that noise-induced $\mathrm{CP}$ plasticity extends to A1 and VAF, but not AAF.

It is possible that not every sound-exposed rat will exhibit CP-like plasticity. However, if $7 \mathrm{kHz}$ map expansion is a reliable indicator, it could be used to distinguish rats that show phenotypic CP plasticity from those that do not. We explored this possibility using a linear discriminant analysis to test the hypothesis that exposed and naive rats could be distinguished based on a linear combination of the $7 \mathrm{kHz}$ percent map areas in more than one auditory field (Figure 1C). Only animals with full maps in both $\mathrm{A} 1$ and VAF were included (7 Naïve and 8 Exposed). The $7 \mathrm{kHz}$ percent map area in A1 and VAF were positively correlated, $r=0.53, p=0.0408$, $n=15$ rats (Figure 1C, top). This is in contrast to $\mathrm{A} 1$ and AAF, $r=0.09, p=0.8068, n=10$ rats, and VAF and AAF, $r=0.22, p=0.5964, n=8$ rats, which were not significantly correlated. The canonical function resulting from the linear discriminant analysis was statistically significant (canonical correlation $=0.79$, Wilks' Lambda $=0.38, F_{(2,12)}=9.68$, $p=0.0031, n=15$ rats, Figure 1C, bottom). Reclassification of the rats based on the new canonical variable using leaveone-out cross-validation was successful: $88.10 \pm 1.2 \%$ of the rats were correctly classified into their exposure condition. The canonical function was positively correlated with both $7 \mathrm{kHz}$ percent map area in A1, $r=0.99, p<0.0001$, $n=15$ rats, and VAF, $r=0.66, p=0.0069, n=15$ rats. This result was approximately equivalent to performing a linear discriminant analysis using the $7 \mathrm{kHz}$ percent map area in A1 alone and better than using VAF alone. When including only A1, the canonical function was significant (canonical correlation $=0.77$, Wilks' Lambda $=0.41, F_{(1,17)}=24.15$, $p=0.0001, n=19$ rats), cross-validated reclassification led to $89.47 \pm 0.41 \%$ correct classification. When including only VAF, the canonical function was also significant but less successful (canonical correlation $=0.53$, Wilks' Lambda $=0.72$, $F_{(1,14)}=5.38, p=0.0360, n=16$ rats). Cross-validated reclassification led to $71.25 \pm 1.4 \%$ correct classification. These results show that the degree of map expansion is relatively consistent within each animal; rats with high map expansion in A1 are likely to have high map expansion in VAF. This characteristic also allows rats that have undergone sound exposure to be classified with high accuracy, suggesting that degree of map expansion is a reliable indicator of $\mathrm{CP}$ plasticity whether taking into account only $\mathrm{A} 1$ or $\mathrm{A} 1$ and VAF together.

To establish the electrophysiological correlates of $7 \mathrm{kHz}$ map expansion, we continued to compare neural response properties in five CF bins using data from both full and partial maps. We predicted that the $7 \mathrm{kHz}$-tuned neurons of exposed animals would show additional evidence of plasticity. We compared the receptive field bandwidth $20 \mathrm{~dB}$ above threshold (BW20), a measure of response specificity, in each auditory field (Figure 1E). In A1, we observed no significant change in BW20 following exposure for any CF bin (mixed-effects two-way ANOVA with Group and Bin as factors. Interaction $F_{(4,687.7)}=1.10, p=0.3545$. Main effect of Group $F_{(1,70.18)}=0.26$, $p=0.6093, n=701$ sites within 52 positions and 23 rats). In AAF we found significantly narrower BW20s for the $7 \mathrm{kHz}$ and $38 \mathrm{kHz}$ bins (mixed-effects two-way ANOVA with Group and Bin as factors. Interaction $F_{(4,278.1)}=3.32, p=0.0113$ followed by simple main effects for $7 \mathrm{kHz} F_{(1,194.5)}=6.25, p=0.0132$ and $38 \mathrm{kHz} F_{(1,127.9)}=11.94, p=0.0007$. No other CF bin was significant $F_{(1,160.3-234.5)} \leq 1.42$, all $p \geq 0.2360, n=292$ sites within 47 positions and 22 rats). In VAF, on the other hand, we observed broader BW20s for the $7 \mathrm{kHz}$ bin (mixed-effects two-way ANOVA with Group and Bin as factors. Interaction $F_{(4,327.1)}=2.74, p=0.0288$, followed by simple main effects for $7 \mathrm{kHz} F_{(1,242.8)}=5.42, p=0.0207$. No other CF bin was significant $F_{(1,121.5-278.7)} \leq 2.78$, all $p \geq 0.0966, n=342$ sites within 45 positions and 23 rats). These differences demonstrate a reduction in tuning specificity for VAF neurons tuned to $7 \mathrm{kHz}$ following sound exposure and an increase in specificity for AAF neurons tuned to $7 \mathrm{kHz}$ and $38 \mathrm{kHz}$.

Cortical thresholds measure a neuron's sensitivity to low intensity sounds and can provide an approximate estimate of hearing thresholds. We compared the average cortical thresholds of neurons in each $\mathrm{CF}$ bin between experimental groups (Figure 1F). We observed no group differences in $\mathrm{A} 1$ or VAF for any CF bin (mixed-effects two-way ANOVAs with Group and Bin as factors A1: Interaction $F_{(4,657)}=1.28$, $p=0.2761$. Main effect of Group $F_{(1,49.79)}=0.01, p=0.9190$, $n=701$ sites within 52 positions and 23 rats. VAF: interaction $F_{(4,310.9)}=0.71, p=0.5867$. Main effect of Group $F_{(1,43.54)}=0.02, p=0.8924, n=342$ sites within 45 positions and 23 rats). In AAF, however, we found that average thresholds were significantly lower for the $7 \mathrm{kHz}$ bin (mixed-effects two-way ANOVA with Group and Bin as factors. Interaction $F_{(4,255.6)}=2.41, p=0.0494$ followed by simple main effects for $7 \mathrm{kHz}\left(F_{(1,124.8)}=4.70, p=0.0320\right.$. No other CF bin was significant $F_{(1,67.93-156.3)} \leq 2.28, p \geq$ $0.1334, n=292$ sites within 47 positions and 22 rats). These results show that after sound exposure, AAF became more sensitive to the $7 \mathrm{kHz}$ frequency. Importantly, the cortical thresholds of the Exposed group were either the same or lower than Naïve for all fields, demonstrating that the exposure intensities were non-traumatic and did not cause any apparent hearing loss. Taken together, the changes in BW20 and cortical thresholds observed in sound-exposed animals may highlight differences in the receptive field properties of AAF and VAF. VAF neurons tend to have narrow tuning curves with low thresholds while AAF neurons tend to have broad tuning curves with relatively high thresholds. Plasticity following sound exposure appears to have reduced these field-specific qualities for $7 \mathrm{kHz}$-tuned neurons.

Sound-evoked firing rates are elevated in hyperacusis (Sun et al., 2012; Aazh et al., 2014; Hickox and Liberman, 2014). We compared the tone-evoked firing rate between exposed 

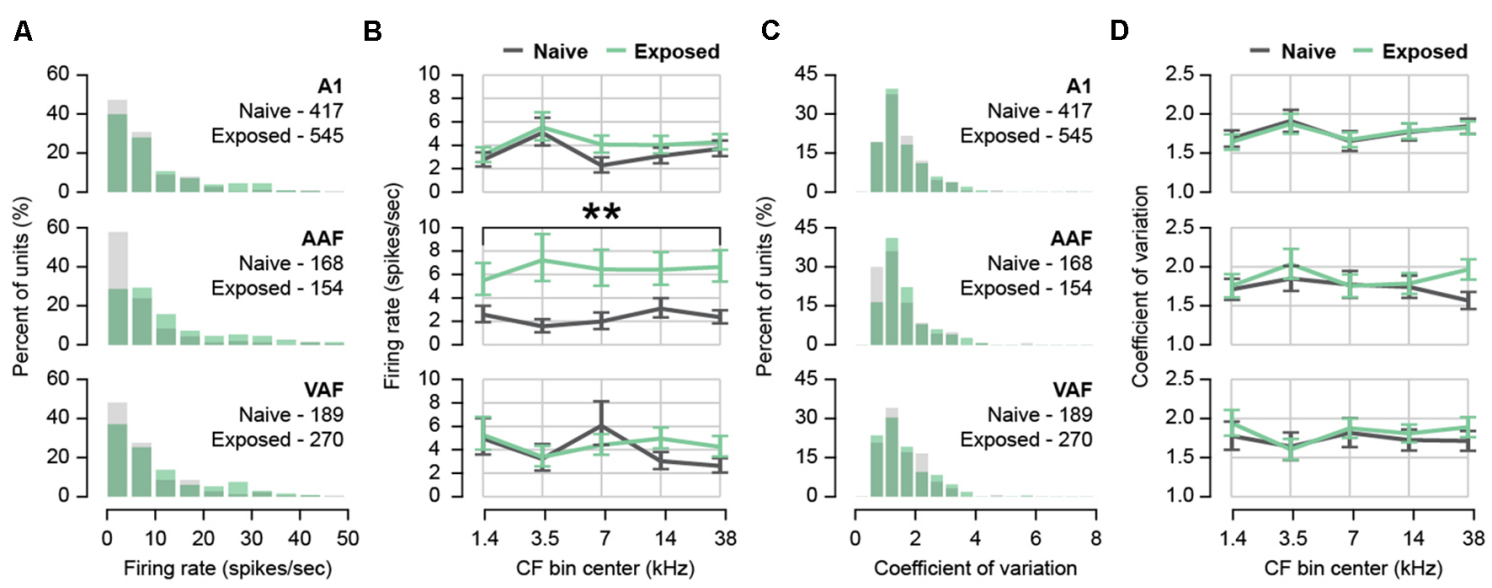

FIGURE 2 | Effect of sound exposure on spontaneous firing rate and burst firing. (A) Histogram of firing rates for each auditory field. N units per field and group in inset. (B) Back-transformed mean firing rate with CF in five frequency bins for each auditory field. (C) Histogram of coefficient of variation (CV) for each auditory field. $\mathrm{N}$ units per field and group in inset. (D) Back-transformed mean CV in five frequency bins for each auditory field. ${ }^{* *} p<0.01$. Error bars represent SEM. A1, primary auditory field; AAF, anterior auditory field; VAF, ventral auditory field. See Table $\mathbf{1}$ for number of rats, recording positions, and units per auditory field and group.

and naive animals (Figure 1G). The average firing rate in response to the full range of tonal stimuli (66 frequencies presented at eight intensities) was considered. The rate was computed from the number of spikes counted between 8 and $58 \mathrm{~ms}$ after tone presentation minus the number of spikes counted in the $50 \mathrm{~ms}$ preceding tone presentation. As could be expected, firing rate was positively correlated with sound intensity, $r=0.11, p<0.0001, n=7,093$ observations. We also found that onset latency was negatively correlated with firing rate, $r=-0.20, p<0.0001, n=7,093$ observations, possibly because a fixed epoch window resulted in less spikes being counted for sites with later latencies. We did not observe a significant difference in onset latency between naïve and exposed animals for any field (mixed effects two-way ANOVAs with Group and Bin as factors. A1: Interaction $F_{(4,678.3)}=1.74$, $p=0.1403$. Main effect of Group $F_{(1,64.63)}=0.39, p=0.5325$, $n=701$ sites within 52 positions and 23 rats. AAF: interaction $F_{(4,253.2)}=1.74, p=0.1414$. Main effect of Group $F_{(1,34.73)}=3.00$, $p=0.0920, n=292$ sites within 47 positions and 22 rats. VAF: interaction $F_{(4,317.9)}=0.13, p=0.9698$. Main effect of Group $F_{(1,37.34)}=0.09, p=0.7683, n=342$ units within 45 positions and 23 rats). As a result, we performed two-way ANCOVAs with intensity and latency as covariates to determine whether the tone-evoked firing rate differed between experimental groups controlling for these two variables. We did not find a significant difference between groups for any CF bin in A1 or VAF (mixed effects two-way ANCOVAs with Group and Bin as factors. A1: interaction $F_{(4,3662)}=0.35, p=0.8456$. Main effect of Group $F_{(1,50.68)}=0.01, p=0.9073, n=3,697$ observations within 52 positions and 23 rats. VAF: interaction $F_{(4,1937)}=1.38$, $p=0.2393$. Main effect of Group $F_{(1,40.82)}=0.10, p=0.7516$, $n=1,965$ observations within 45 positions and 23 rats). In AAF, however, we observed a significantly higher tone-evoked firing rate for the $3.5 \mathrm{kHz}, 7 \mathrm{kHz}$, and $38 \mathrm{kHz}$ bins (mixed effects two-way ANCOVAs with Group and Bin as factors. Interaction
$F_{(4,1398)}=9.02, p<0.0001$ followed by simple main effects for $3.5 \mathrm{kHz} F_{(1,60.06)}=4.39$, p $0.0403,7 \mathrm{kHz} F_{(1,67.83)}=4.99$, $p=0.0288$, and $38 \mathrm{kHz} F_{(1,47.66)}=8.03, p=0.0067$. No other CF bins were significant $F_{(1,51.18-60.75)} \leq 0.23$, both $p \geq 0.63$, $n=1,431$ observations within 47 positions and 22 rats) For all of the ANCOVAs above, intensity $\left(F_{(1,1383-3646)} \geq 35.56\right.$, all $p \leq 0.0001)$ and latency $\left(F_{(1,1406-3666)} \geq 78.36\right.$, all $\left.p \leq 0.0001\right)$ remained significant factors. These results show that after sound exposure, tone-evoked firing rate was greater within AAF for neurons tuned to a broad range of frequencies.

Tinnitus and hyperacusis are associated with higher spontaneous firing rates (Wang et al., 2011; Kaltenbach, 2011), and tinnitus in particular is associated with more burst firing in the auditory pathway including the auditory thalamus (Kalappa et al., 2014) and auditory cortex (Syka and Rybalko, 2000; Noreña and Eggermont, 2003). From 5-min-long recordings of spontaneous activity during silence, we computed the spontaneous firing rate and inter-spike intervals (ISIs) of single-unit activity (Figure 2). Each sorted unit was assigned an auditory field and CF based on sound-evoked responses in the same recording position resulting in a total of 1,743 units from A1, AAF, and VAF combined. The number of units included in each auditory field and group is listed in Table $\mathbf{1 .}$ Because the distribution of firing rates was positively skewed (Figure 2A), we applied a natural logarithmic transform before statistical analyses. Back-transformed means are plotted in Figure 2B. The average spontaneous firing rates of our naïve animals were as follows: $\mathrm{A} 1=5.33 \pm 0.33, \mathrm{AAF}=4.77 \pm 0.58$, $\mathrm{VAF}=5.67 \pm 0.57$ spikes/second. After sound exposure, we did not observe any difference in spontaneous firing rates in $\mathrm{A} 1$ or $\mathrm{VAF}$ regardless of $\mathrm{CF}$ bin (mixed effects two-way ANOVAs with Group and Bin as factors. A1: Interaction $F_{(4,932.2)}=0.85, p=0.4951$. Main effect of Group $F_{(1,40.85)}=1.04$, $p=0.3134, n=962$ units within 44 positions and 23 rats. VAF: Interaction $F_{(4,442.9)}=1.65, p=0.1596$. Main effect of Group 
$F_{(1,37.86)}=0.34, p=0.5626, n=459$ units within 37 positions and 22 rats). In AAF, on the other hand, sound exposure led to a significant and uniform increase in firing rate for all CF bins (mixed effects two-way ANOVA with Group and Bin as factors. Interaction $F_{(4,304.7)}=1.06, p=0.3759$. Main effect of Group $F_{(1,39.57)}=14.67, p=0.0004, n=322$ units within 37 positions and 22 rats). The increased spontaneous firing rate in AAF indicates strong, tuning-independent hyperactivity resulting from sound exposure.

The ISI coefficient of variation (CV) was used to estimate the bursting activity of auditory neurons. This measure was obtained by dividing the standard deviation of each unit's ISI distribution by its mean (Longenecker and Galazyuk, 2016). A high CV indicated more irregular spiking intervals, suggestive of bursting. Again, the distribution of CVs was positively skewed (Figure 2C) so a natural logarithmic transform was applied before statistical analyses and back-transformed means are plotted in Figure 2D. We did not observe any difference in the average CV of any field after sound exposure (mixed effects two-way ANOVAs with Group and Bin as factors. A1: Interaction $F_{(4,932.3)}=0.07$, $p=0.9908$. Main effect of Group $F_{(1,39.63)}=0.01, p=0.9126$, $n=962$ units within 44 positions and 23 rats. AAF: Interaction $F_{(4,306.8)}=1.40, p=0.2325$. Main effect of Group $F_{(1,36.99)}=0.78$, $p=0.3842, n=322$ units within 37 positions and 22 rats. VAF: Interaction $F_{(4,433.8)}=0.32, p=0.8627$. Main effect of Group $F_{(1,33)}=0.30, p=0.5886, n=459$ units within 37 positions and 22 rats). From this, we concluded that burst firing was unchanged in the auditory cortex following sound exposure.

Tinnitus has also been associated with hypersynchronization in animal models. Hypersynchronization typically appears immediately after noise trauma in a frequency-specific manner (Eggermont and Roberts, 2004) and is evidence of increased connectivity, either thalamocortical or corticocortical, between neurons. To assess whether the Exposed group displayed hypersynchronization, we calculated normalized cross-correlograms between single-unit pairs recorded in silence (Figure 3). From the 1,743 units detected above, we identified 16,441 unit pairs in separate channels. We limited our analysis to pairs with a peak between -150 and $150 \mathrm{~ms}$, falling within approximately \pm 2.3 standard deviations of the mean peak, resulting in a total of 14,008 unit pairs for all fields. Figure 3A shows histograms of the cross-correlogram peak lag times demonstrating that peaks tend to fall near $0 \mathrm{~ms}$ and Figure 3B shows the average cross-correlogram for all pairs in each field. The peak value of the cross-correlogram tended to decrease with greater inter-unit distance, $r=-0.24, p<0.0001$, $n=14,008$ pairs, as well as greater $\Delta \mathrm{CF}, r=-0.24, p<0.0001$, $n=14,008$ pairs. Distance and $\Delta \mathrm{CF}$ were positively related, $r=0.42, p<0.0001, n=14,008$ pairs. As a result, we performed mixed-effects two-way ANCOVAs with distance as a covariate to determine whether the peak correlation coefficient differed between exposure groups while controlling for differences in inter-unit distance (Figure 3C). As the distribution of peaks was positively skewed, we multiplied the data, originally on a $0-1$ scale, by 100 and applied a natural logarithmic transform before statistical analyses. In Figure 3C, the difference between back-transformed group means for each CF bin combination is depicted by a heatmap. The interaction was significant for A1, AAF, and VAF (mixed effects two-way ANCOVAs with Group and Combined CF Bin as factors and Distance as covariate: A1 $F_{(24,9632)}=5.35, p<0.0001, \operatorname{AAF} F_{(24,1407)}=2.16, p=0.0009$, VAF $\left.F_{(24,2730)}=1.88, p=0.0060\right)$. The simple main effect of Group was evaluated over each level of Combined CF Bin and the significant comparisons are outlined in bold on the heatmap in Figure 3C. Distance remained a significant covariate in each ANCOVA $\left(\mathrm{A} 1 F_{(1,9650)}=358.29, p<0.0001, \mathrm{AAF}\right.$ $\left.F_{(1,1406)}=47.43, p<0.0001, \operatorname{VAF} F_{(1,2740)}=47.48, p<0.0001\right)$. From the heatmaps, we observed few significant differences in synchronization strength. Sound-exposed A1 and VAF tended to have shorter cross-correlograms for most frequency combinations, with peak values being significantly smaller for low-to-medium frequency combinations only. In AAF, differences with respect to naïve animals were less consistent. Only synchronization between unit pairs where both units had CFs in the $38 \mathrm{kHz}$ bin was significantly greater.

The strength of synchronization can also be estimated by the width of the cross-correlogram, with wider functions representing greater synchronization at longer lag times. The width at half-height of each peak was compared between exposure groups as a function of CF bin (Figure 3D). Width could not be computed for 38 pairs for which the function did not dip below half-height, resulting in 13,970 analyzed pairs. Width was found to weakly but significantly increase with inter-unit distance, $r=0.03, p=0.0009, n=13,970$ pairs, and $\Delta \mathrm{CF}$, $r=0.03, p=0.0013, n=13,970$. However, distance did not remain significant when included as a covariate for any field (mixed effects two-way ANCOVAs with Group and Combined $\mathrm{CF}$ Bin as factors and Distance as covariate. Effect of Distance: A1 $F_{(1,9651)}=0.22, p=0.6381, \operatorname{AAF} F_{(1,1403)}=0.003, p=0.9545$, $\left.\operatorname{VAF} F_{(1,2739)}=0.41, p=0.5239\right)$. As a result, we removed the covariate and performed mixed-effects two-way ANOVAs. The interaction was significant for A1, AAF, and VAF (mixed effects two-way ANOVA with Group and Combined CF Bin as factors: A $1 F_{(24,9499)}=4.12, p<0.0001, \operatorname{AAF} F_{(24,1422)}=2.60, p<0.0001$, VAF $\left.F_{(24,2741)}=1.67, p=0.0214\right)$. The simple main effect of Group was evaluated over each level of Combined CF Bin and the significant comparisons are outlined in bold on the heatmap in Figure 3D. In the heatmaps, we observed clear wider crosscorrelograms in the sound-exposed A1, AAF, and VAF. In A1, this trend showed units in low-to-mid frequency bins having wider cross-correlograms with units in the highest frequency bins. In AAF, almost every frequency bin combination tended to have wider cross-correlograms, with significant differences in the mid-to-high frequency combinations, and notably with the $7 \mathrm{kHz}$ bin showing the greatest increase in width. Interestingly, VAF showed an opposite trend, where only the lowest frequency bins had significantly wider cross-correlograms when paired with the highest frequency bins. The mid-range bins, including $7 \mathrm{kHz}$, showed either no change in width or a slight decrease in width for VAF.

A greater average cross-correlogram width could result from either more pairs with broad cross-correlograms or more pairs with off-centered peaks. To investigate the contribution of pairs with off-centered peaks to the wider cross-correlograms we 

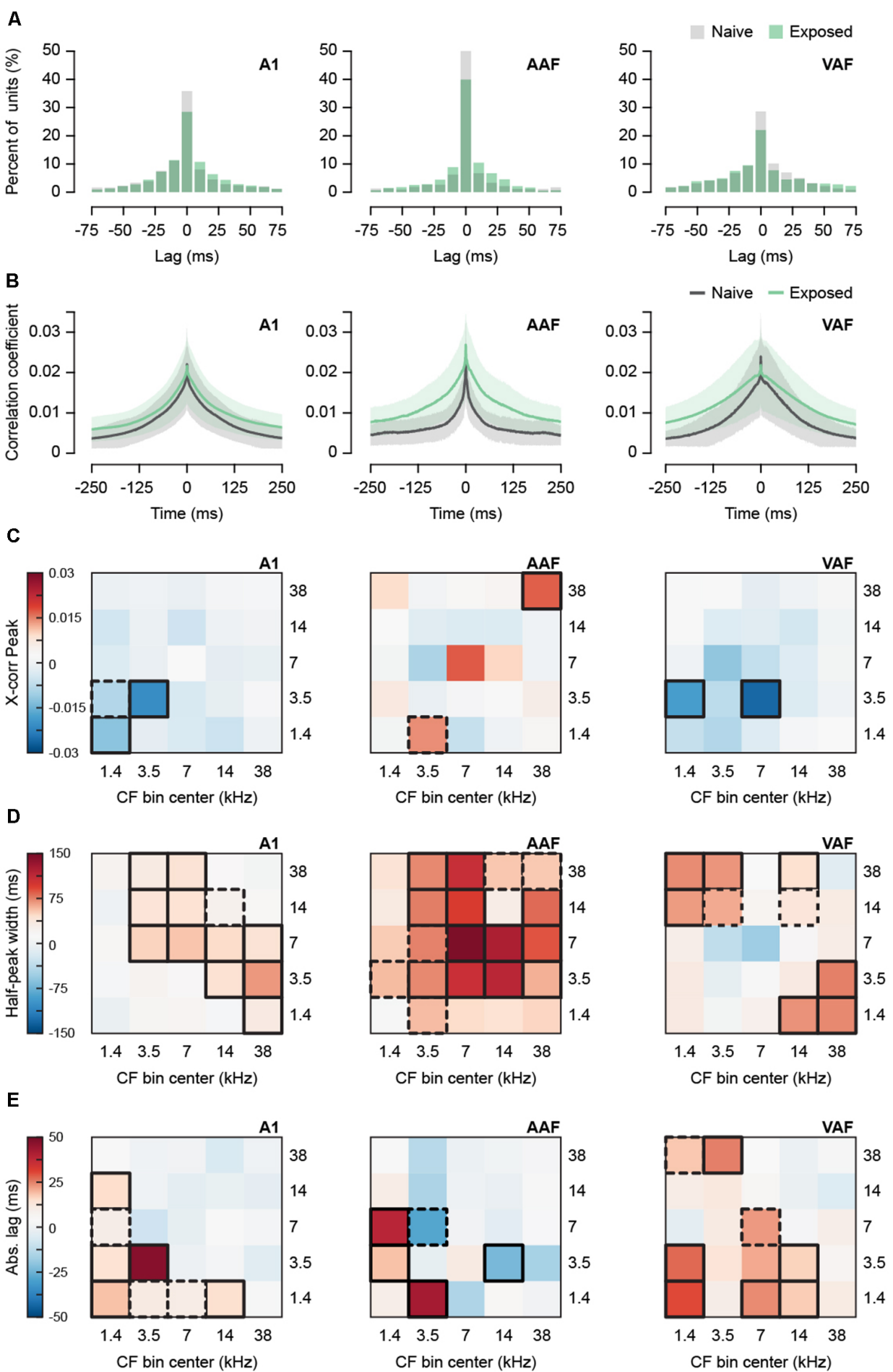

FIGURE 3 | Effect of sound exposure on spontaneous synchronization. (A) Histogram of lag times of the peak of the cross-correlogram for all recorded unit pairs in A1, AAF, and VAF. Data for lag times outside -75 and 75 are not shown. (B) Average cross-correlogram for all unit pairs detected in separate channels in A1, AAF, and VAF. Shaded region represents SEM. (C) Subtracted (Exposed-Naïv) difference between average peak correlation coefficient for unit pairs with CF in five frequency bins. (D) Subtracted (Exposed-Naïve) difference between average half-peak width for unit pairs with CF in five frequency bins. (E) Subtracted (Exposed-Naïve) difference between average time lag in absolute values of the peak of the cross-correlogram. Bolded boxes are significant with $p<0.05$. Dashed boxes represent $p$-values < 0.10. A1, primary auditory field; AAF, anterior auditory field; VAF, ventral auditory field; CF, characteristic frequency. $N$ unit pairs per auditory field and group: Naïve A1 3,614; AAF 746; VAF 1,092. Exposed A1 6,116; AAF 733; VAF 1,707. See Table 1 for number of rats, recording positions, and units per auditory field and group. 
observed in each field, we conducted mixed-effects two-way ANCOVAs with distance as a covariate on the absolute lag of the peak of the cross-correlogram (Figure 3E). Distance was positively correlated with absolute lag, $r=0.23, p<0.0001$ $n=14,008$ pairs. The interaction between exposure group and CF bin was significant for A1, AAF, and VAF (mixed effects two-way ANCOVA with Group and Combined CF Bin as factors and Distance as covariate: $\mathrm{A} 1 F_{(24,9499)}=4.12, p<0.0001$, AAF $\left.F_{(24,1422)}=2.60, p<0.0001, \operatorname{VAF} F_{(24,2741)}=1.67, p=0.0214\right)$. The simple main effect of Group was evaluated over each level of Combined CF Bin and the significant comparisons are outlined in bold on the heatmap in Figure 3E. Distance remained a significant covariate for all three fields $\left(\mathrm{A} 1 F_{(1,9679)}=305.61\right.$, $p<0.0001, \operatorname{AAF} F_{(1,1427)}=7.50, p=0.0062, \operatorname{VAF} F_{(1,2748)}=45.29$, $p<0.0001)$. The heatmaps revealed mostly increases in absolute lag for the Exposed group, suggestive of a greater number of off-centered peaks. However, in A1 and AAF, the CF bins with greater absolute lag did not correspond with those that showed the broadest widths in Figure 3D. This suggests that a greater number of broadly synchronized unit pairs contribute to the wider cross-correlograms in these fields. In VAF, some CF bins with wider cross-correlograms corresponded with bins that also had greater absolute lag, indicating a mixed contribution between broader synchronization and off-centered peaks.

\section{Behavioral Correlates of Sound Exposure}

A common behavioral measure for detecting tinnitus in rodents is gap-prepulse inhibition of the acoustic startle response (GPIAS), in which a short silent gap within a background sound carrier reduces the magnitude of a rodent's involuntary startle to a subsequent loud noise burst (Brozoski and Bauer, 2016). Impaired GPIAS is considered evidence of tinnitus in rodents, meaning that the gap is less effective at reducing the startle response, possibly because the presence of tinnitus interferes with the ability to hear silence. This test is usually accompanied by a similar measure called prepulse inhibition (PPI) of the acoustic startle response. PPI has been proven useful in characterizing hyperacusis and hypoacusis, since a short tonal stimulus will either enhance or dampen inhibition of the startle response in rodents with hyper- or hypoacusis, respectively (Carlson and Willott, 1996; Turner and Parrish, 2008; Turner and Larsen, 2016; Pienkowski, 2018). To investigate whether sound exposure could have altered these behavioral measures, we performed GPIAS and PPI testing on two additional groups of naïve (Naïve$\mathrm{BEH}, N=12$ ) and exposed (Exposed-BEH, $N=12$ ) rats.

We found that the Exposed-BEH group did not differ from Naïve-BEH in GPIAS (Figure 4). A schematic of the behavioral protocol for GPIAS is presented in Figure 4A. We hypothesized that a deficit in inhibition of the acoustic startle response would be specific to the $7 \mathrm{kHz}$ exposure frequency. To test this, we performed testing in the presence of either a $7 \mathrm{kHz}$ pure tone background or a $3.5 \mathrm{kHz}$ pure tone background with the order of testing counterbalanced between pairs. First, we confirmed that the magnitude of the response to the startle pulse alone was not significantly different between groups for either pure tone condition $\left(7 \mathrm{kHz}\right.$ : two-tailed paired $t$-test $t_{(11)}=-0.81$, $p=0.4372$, Cohen's dav $-0.32 ; 3.5 \mathrm{kHz}$ : two-tailed paired $t$-test $t_{(11)}=-0.08, p=0.9371$, Cohen's dav $-0.03, n=12$ pairs, Figures 4B,C, bottom left). Next, we computed the percent reduction in the startle response when the startle pulse was preceded by a silent gap. We found that the average reduction in startle did not differ between groups for either the $7 \mathrm{kHz}$ (one-tailed paired $t$-test $t_{(11)}=-0.45 p=0.3303$, Cohen's dav $-0.22, n=12$ pairs, Figure 4B) or the $3.5 \mathrm{kHz}$ (one-tailed paired $t$-test $t_{(11)}=0.44 p=0.6656$, Cohen's dav $0.20, n=12$ pairs, Figure 4C) condition. From these results, we concluded that sound exposure did not lead to behavioral evidence of tinnitus in any frequency tested.

We observed an enhancement in PPI for the Exposed-BEH group when the prepulse was a $7 \mathrm{kHz}$ tone (Figure 5). A schematic of the behavioral protocol for PPI is presented in Figure 5A. Of note, a magnified response to the startle pulse alone is also sometimes taken as evidence of hyperacusis (Chen et al., 2013), but we hypothesized that an improvement in inhibition of the acoustic startle response would be specific to the $7 \mathrm{kHz}$ exposure frequency. As a result, we performed PPI testing using either a $7 \mathrm{kHz}$ or $3.5 \mathrm{kHz}$ pure tone prepulse with the order of $7 \mathrm{kHz}$ prepulse, $3.5 \mathrm{kHz}$ prepulse, and no prepulse trials randomly interleaved within a single testing session. Testing took place in the presence of a $65 \mathrm{~dB}$ white noise background. We observed that the magnitude of the response to the startle pulse alone was not significantly different between groups (paired $t$-test $t_{(11)}=-0.21, p=0.8343$, Cohen's dav $-0.09, n=12$ pairs, Figure 5B, bottom left). Next, we compared the average percent reduction in the startle response when the startle pulse was preceded by a prepulse tone. We found that the average reduction in startle was significantly greater for the Exposed-BEH group when the prepulse was a $7 \mathrm{kHz}$ tone (onetailed paired $t$-test $t_{(11)}=2.69 p=0.0105$, Cohen's dav 0.63, $n=12$ pairs, Figure 5B) but not when the prepulse was a $3.5 \mathrm{kHz}$ tone (one-tailed paired $t$-test $t_{(11)}=0.66 p=0.2621$, Cohen's dav $0.29, n=12$ pairs, Figure 5C) condition. Our positive findings remained significant when adjusting the alpha value to account for three comparisons using either the Bonferroni or HolmsBonferroni correction (both $\alpha=0.0167$ ). From these results, we concluded that Exposed-BEH exhibited behavioral evidence of hyperacusis for the $7 \mathrm{kHz}$ frequency.

\section{DISCUSSION}

Passive exposure to moderate-intensity broadband white noise can be used to open a CP window for frequency tuning in the adult rat auditory cortex, allowing for subsequent frequency-specific reorganization of the tonotopic map. This phenomenon could have profound implications for plasticitybased neurotherapeutics that aim to improve learning and memory or treat disorders of plasticity through non-invasive means. However, frequency-specific tonotopic map expansions and regional changes in excitability have also been described as symptoms of tinnitus and hyperacusis in animal models, leading us to wonder whether noise exposure could increase the risk of developing one or both of these disorders. In the present investigation, we extended previous studies by examining the effects of noise and tone pip exposure on secondary 

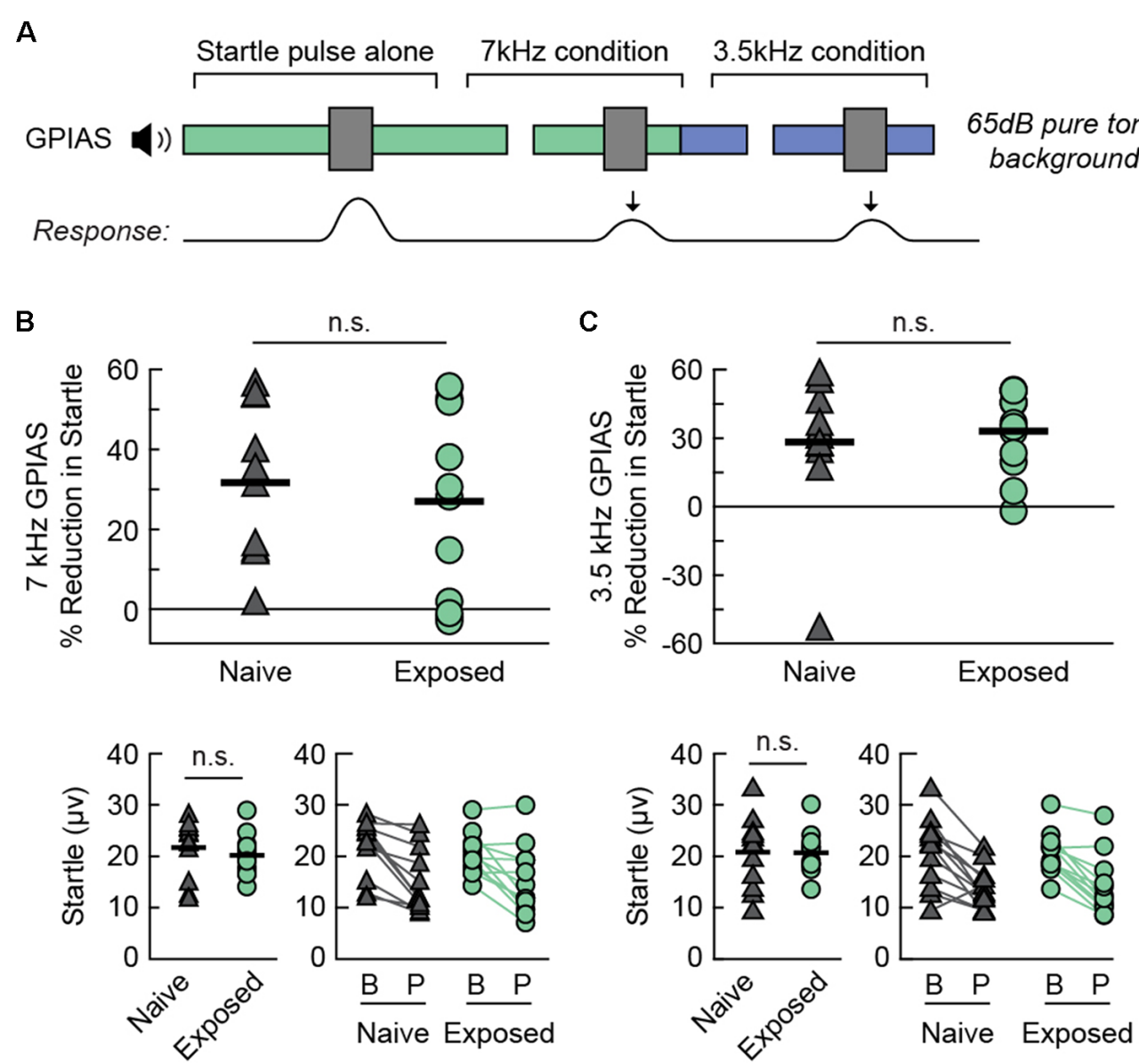

FIGURE 4 | Sound exposed rats demonstrate no change in GPIAS. (A) A schematic drawing of the behavioral protocol. Testing takes place in the presence of a $65 \mathrm{~dB}$ SPL continuous pure tone ( 3.5 or $7 \mathrm{kHz}$ ). A $30 \mathrm{~ms}$ silent gap prepulse preceding the startle sound (40 ms white noise burst, $120 \mathrm{~dB}$ SPL) reduces the magnitude of the acoustic startle response. (B) Percent GPIAS (Top), baseline startle response (Bottom Left), and comparison of startle response between Baseline (B) and Prepulse (P) trials (Bottom Right) in the presence of a $7 \mathrm{kHz}$ pure tone background. Lines connect responses from the same animal. (C) Percent GPIAS (Top), baseline startle response (Bottom Left), and comparison of startle response between Baseline (B) and Prepulse (P) trials (Bottom Right) in the presence of a $3.5 \mathrm{kHz}$ pure tone background. Lines connect responses from the same animal. Ns, not significant. N rats per group: 12 Naive, 12 Exposed. GPIAS, Gap-Prepulse Inhibition of the Acoustic Startle response.

auditory fields and carried out novel experiments to determine whether sound-exposed animals display evidence of tinnitus or hyperacusis.

As in previous studies (Zhou et al., 2011; Thomas et al., 2018), we observed map expansion in the A1 of adult rats passively exposed to moderate-intensity broadband white noise followed by tone pips with no elevation in cortical thresholds. We also showed for the first time that a CP-like window is also opened in VAF as demonstrated by map expansion in this field accompanied by broader receptive field bandwidths for $7 \mathrm{kHz}$-tuned neurons. Apart from map expansion, however, we observed few changes in spontaneous activity or auditory processing in the $\mathrm{A} 1$ and $\mathrm{VAF}$ of exposed animals. In contrast, we observed strong evidence of hyperactivity in AAF, where there was no map expansion. This included an overall increased spontaneous firing rate, stronger tone-evoked firing rates and narrower receptive field bandwidths for a range of frequencies, and a lower cortical threshold for $7 \mathrm{kHz}$-tuned neurons. Despite changes in AAF affecting multiple iso-frequency bands, the band corresponding to $7 \mathrm{kHz}$ showed changes consistent with heightened sensitivity in all of our measures. Our behavioral results also pointed to enhanced sensorimotor gating for the $7 \mathrm{kHz}$ frequency, since exposed rats had improved PPI when the prepulse was a $7 \mathrm{kHz}$ pure tone. Taken together, our findings point to hyperacusis for the $7 \mathrm{kHz}$ frequency in soundexposed animals.

We expected hypersynchronization to accompany map expansion given the close link between receptive field overlap and neural synchronization (Noreña and Eggermont, 2006; Eggermont, 2007; Kilgard et al., 2007). However, we did not observe clear hypersynchronization in any field. The absence of this relationship could be due to the unique manner in which noise induces plasticity. Noise exposure on its own produces lasting desynchronization with shorter cross-correlogram peaks 

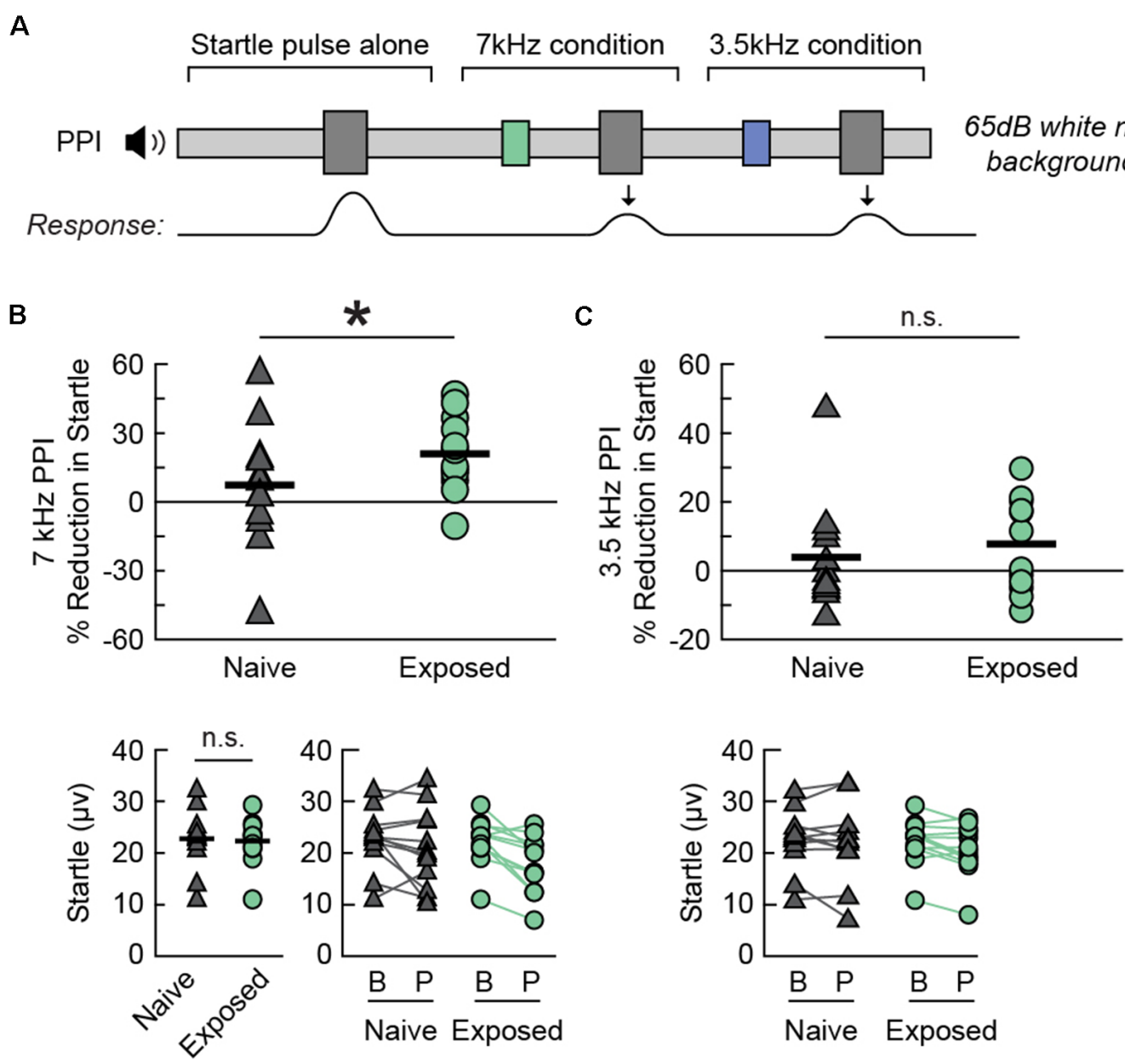

FIGURE 5 | Sound exposed rats demonstrate a frequency-specific enhancement in PPI. (A) A schematic drawing of the behavioral protocol. Testing takes place in the presence of a continuous $65 \mathrm{~dB}$ SPL background noise. A 20 ms tone pip ( 3.5 or $7 \mathrm{kHz}, 75 \mathrm{~dB}$ SPL) preceding the startle sound (40 ms white noise burst, $120 \mathrm{~dB}$ SPL) reduces the magnitude of the acoustic startle response. (B) Percent PPI (Top), baseline startle response (Bottom Left), and comparison of startle response between Baseline $(B)$ and Prepulse $(\mathrm{P})$ trials (Bottom Right) when the prepulse is a $7 \mathrm{kHz}$ tone. Lines connect responses from the same animal. (C) Percent $\mathrm{PPI}$ (Top) and comparison of startle response between Baseline (B) and Prepulse (P) trials (Bottom) when the prepulse is a $3.5 \mathrm{kHz}$ tone. Lines connect responses from the same animal. Note that the same baseline startle values were used for computing PPI in (B,C). ${ }^{*} p<0.05$, ns, not significant. $N$ rats per group: 12 Naive, 12 Exposed. PPI, Prepulse Inhibition of the acoustic startle response.

in A1 (Zhou et al., 2011; Kamal et al., 2013; Thomas et al., 2018). The prevalence of lower peaks and broader cross-correlogram widths observed in A1 and VAF could be consistent with these earlier findings, assuming partial recovery of desynchronization potentially hastened by tone pip exposure. The broader widths that we observed in AAF centered on $7 \mathrm{kHz}$ are likely the combined result of greater disinhibition and increased firing, since secondary effects independent of connectivity can also affect the width of the cross-correlogram. These include firing patterns intrinsic to each neuron, such as burst firing, and global oscillations (Eggermont and Smith, 1996; Nowak and Bullier, 2005). Although cross-correlograms were normalized with respect to firing rate and significant differences in burst firing measured by $\mathrm{CV}$ were not observed, a higher firing rate could increase the impact of secondary effects on cross-correlogram width. Clear evidence of disinhibition or changes in firing were not observed in A1 or VAF in the present study, therefore assumptions about the origin of broader crosscorrelogram widths beyond residual effects of noise exposure remain speculative.

The role of the auditory cortex in generating tinnitus and hyperacusis has not been fully established. Although changes in neural activity related to hearing loss begin in the auditory nerve and cochlear nucleus, individuals with clinically normal audiograms can also report these percepts, and electrophysiological signatures of each condition have been reported in cortex in animal models. In addition, studies have primarily identified A1 and the auditory thalamus (medial geniculate body, MGB) as sites of experience-dependent plasticity following non-traumatic passive sound exposures (Pienkowski and Eggermont, 2011; Lau et al., 2015; Pienkowski, 2018), revealing a possible mechanism by which passive 
experience could lead to changes in auditory processing in the absence of hearing loss. Here, we observed a significant difference between the electrophysiological response properties of A1 and AAF following noise exposure that may suggest a causal role for AAF in the generation of hyperacusis. Whereas A1 has been studied extensively in the context of passive sound exposure, much less is known about how AAF adapts to such experiences. Sparse findings demonstrate asymmetric plasticity in each field despite both receiving direct inputs from the ventral MGB and displaying similar tone-evoked response properties (Polley et al., 2007). Takahashi et al. (2006) found that A1 responses of juvenile mice were more potentiated than those of AAF following exposure to an amplitude-modulated tone for 4-5 weeks but did not observe an over-representation of the exposure frequency in either field. A recent study documented differences in parvalbumin positive $(\mathrm{PV}+)$ interneuron and peri-neuronal net $(\mathrm{PNN})$ densities in $\mathrm{A} 1$ and AAF following $70 \mathrm{~dB}$ SPL broadband noise exposure in mice during the first month of life (Reinhard et al., 2019). Noise exposure decreased the density of PNNs in A1 but not AAF, showing that inhibitory elements can be differently regulated across these two fields. Given the preliminary nature of our study, further studies should be undertaken to understand the potential mechanisms by which AAF could contribute to hyperacusis.

The sound exposure paradigm used to induce $7 \mathrm{kHz}$ map expansion consisted of two distinct components: white noise exposure and $7 \mathrm{kHz}$ tone pip exposure. On its own, chronic exposure to moderate-intensity white noise has been shown to lead to tonotopic disorganization, broadened receptive field bandwidths, decreased neural synchronization, and disrupted temporal processing. These plastic changes develop whether the noise is present for 2 weeks (Thomas et al., 2018), 6-8 weeks (Zhou et al., 2011; Kamal et al., 2013), or on a 10-h-perday schedule (Zhou and Merzenich, 2012). As long as the noise is broadband, its effects are non-frequency-specific as illustrated by comparison with band-limited noise exposure (de Villers-Sidani et al., 2008). In the present study, the most prominent electrophysiological measure that was affected in a non-frequency-specific manner was the increased spontaneous firing rate in AAF, and it is possible that this change was driven primarily by white noise exposure. Exposure to non-traumatic white noise has been scarcely studied in the context of PPI or GPIAS, especially in contrast to traumatic noise exposures. One exception is a recent study that found that band-limited noise exposure did not produce either hyper- or hypoacusis in mice exposed for 3 months (Pienkowski, 2018).

Taken alone, exposure to pure tones has not been shown to induce strong cortical plasticity leading to map expansion or altered discrimination abilities for the exposure frequency in adult rodents (Zhou et al., 2011; Blundon et al., 2017). This is consistent with the view that the mature cortex is largely resistant to change based on passively experienced stimuli (Keuroghlian and Knudsen, 2007). However, extensive research performed in cat auditory cortex has shown convincingly that band-limited tone pip ensembles can lead to frequency-specific changes in auditory responsiveness after chronic exposure (Pienkowski and Eggermont, 2009, 2010; Pienkowski et al.,
2011). Specifically, cortical regions tuned to the exposure frequency range show reduced responsiveness while cortical regions outside the exposure frequency range show increased responsiveness. This suggests that there could be detectable differences in the electrophysiological properties of the auditory cortex after tone pip exposure that could also alter behavioral responses to PPI or GPIAS. However, extrapolating from these results one would expect animals exposed to $7 \mathrm{kHz}$ to display hypoacusis for this frequency. Consequently, we do not believe that tone pip exposure on its own would cause increased cortical sensitivity to $7 \mathrm{kHz}$.

A limitation of the present study is that in the interest of reducing the number of animals used, the electrophysiological data for the Exposed group came from combining two groups of sound-exposed animals that underwent slightly different $7 \mathrm{kHz}$ exposures (i.e., $7 \mathrm{kHz}$ tone pips vs. $7-8.3 \mathrm{kHz}$ tone pip clouds, see "Materials and Methods" section). It is likely that these exposures would produce different electrophysiological signatures. For example, we would expect tone pip clouds to lead to map expansion for a broader frequency range. To account for this, we used relatively coarse ( $\geq 1$ octave) CF bins in our analysis so that the $7 \mathrm{kHz}$ bin spanned $5-10 \mathrm{kHz}$ and presumably encompassed all neurons that would have shifted their CFs to the exposure frequencies. However, the combined group contained a greater number of rats exposed to tone pip clouds $(n=9)$ than tone pips $(n=4)$, so it is possible that the average data is a better representation of the tone pip cloud exposure. The Exposed-BEH group, on the other hand, was not heterogeneous; every rat was exposed to the tone pip stimulus. As a result, the Exposed group used for electrophysiology is not a perfect analogue for the Exposed-BEH group. Furthermore, because the animals used for behavioral testing in our study were not the same animals that were used for electrophysiological recording, we were unable to correlate auditory response properties with PPI or GPIAS. This additionally prevents us from making any direct conclusions about cortical properties, such as degree of map expansion, that may have influenced inhibition of the acoustic startle response.

A second limitation is that classifying auditory fields based purely on functional characteristics will always result in an imperfect classification. It is possible that some cortical sites, especially those on borders with CF gradient reversals (such as A1 and AAF), were misclassified as being in neighboring fields. Of note, we were unable to distinguish VAF and SRAF based purely on functional properties and therefore pooled the data from these fields. These challenges are not unique to our study, and there is precedence for pooling VAF and SRAF with sparse datasets (Takahashi et al., 2011). Without accompanying anatomical tracer data or similar, conclusions about the response properties of any auditory field should only be drawn from multiple independent replications. Importantly, an experimenter blind to the identity of the experimental groups performed field classification for the present study. The average response properties reported for each field in Supplementary Figure S1 are in strong agreement with the published literature on the adult rat auditory cortex (Polley et al., 2007; Profant et al., 2013).

Finally, stress is another factor that could have played a role in our results, as it is known to affect PPI (Guercio et al., 
2014). Importantly, chronic noise exposure, even at moderate intensities, is a known stressor for humans and animals and has a complex interplay with tinnitus, mostly exacerbating its symptoms (Eggermont, 2017a). The chronic sound exposures used in our study could have caused stress that could affect the acoustic startle response or PPI. The main argument against this, however, is that we did not observe differences in baseline startle response between exposed and unexposed animals. Furthermore, any stress-induced differences in PPI or GPIAS would likely not have been specific to the $7 \mathrm{kHz}$ frequency.

In summary, our study examines the phenomenon of noiseinduced map expansion and demonstrates that prolonged exposure to moderate-intensity noise could be considered a risk factor for hyperacusis in adulthood. Our results could have implications for noise levels presently deemed "safe" in occupational, private, and public settings (Pienkowski and Eggermont, 2012; Gourévitch et al., 2014; Eggermont, 2017b). Rather than suggesting that noise exposure should not be used for neurotherapeutic purposes, however, we would urge continued investigation into this subject. For one, sensorimotor gating measured by PPI is impaired in some neuropsychiatric disorders, most notably schizophrenia (Swerdlow et al., 2000; Swerdlow and Light, 2018). Noise-induced map expansion could thus be a way to target and reverse this specific preattentional deficit (Braff and Light, 2004). Additional candidate strategies to drive plasticity in a sensory-specific manner are vagus nerve stimulation paired with the presentation of pure tones (Engineer et al., 2011) and cognitive training programs designed to improve basic sensory processing (Cramer et al., 2011; Merzenich et al., 2014). Exciting or inhibiting specific brain areas through sensory experience is a more targeted and non-invasive means of driving plasticity than purely pharmaceutical strategies such as those presently used in the treatment of schizophrenia (Guercio et al., 2019) and dementia (Farlow and Cummings, 2007; Massoud and Léger, 2011). By focusing on "retuning" cortical maps, sensory deprivation or stimulation paradigms in other systems could potentially be developed to treat or reverse symptoms of sensory disorders such as phantom sensations or chronic pain (Flor et al., 2001; Tabot et al., 2015). Through both electrophysiological and behavioral measures, the results of our study suggest that map expansion induced by passive sound exposure opens windows of plasticity that can also be understood as windows of vulnerability. However, as our

\section{REFERENCES}

Aarts, E., Verhage, M., Veenvliet, J. V., Dolan, C. V., and van der Sluis, S. (2014). A solution to dependency: using multilevel analysis to accommodate nested data. Nat. Neurosci. 17, 491-496. doi: 10.1038/nn.3648

Aazh, H., McFerran, D., Salvi, R., Prasher, D., Jastreboff, M., and Jastreboff, P. (2014). Insights from the first international conference on hyperacusis: causes, evaluation, diagnosis and treatment. Noise Health 16, 123-126. doi: 10.4103/1463-1741.132100

Adjamian, P., Sereda, M., and Hall, D. A. (2009). The mechanisms of tinnitus: perspectives from human functional neuroimaging. Hear. Res. 253, 15-31. doi: 10.1016/j.heares.2009.04.001

Blundon, J. A., Roy, N. C., Teubner, B. J. W., Yu, J., Eom, T.-Y., Sample, K. J., et al. (2017). Restoring auditory cortex plasticity in adult mice by restricting understanding of the rules that regulate plasticity and the opening and closure of CPs progress, our hope is that we will 1 day be able to harness them to treat a variety of brain disorders.

\section{DATA AVAILABILITY STATEMENT}

The datasets generated for this study can be obtained upon request from the study authors.

\section{ETHICS STATEMENT}

The animal study was reviewed and approved by Montreal Neurological Institute Animal Care Committee.

\section{AUTHOR CONTRIBUTIONS}

GG and EV-S designed the study. GG, KD and MT performed the experiments. GG and $\mathrm{KD}$ obtained the behavioral data. MT obtained the electrophysiological data, analyzed the data, and wrote the first draft of the manuscript. All authors contributed to manuscript revision, read, and approved the submitted version.

\section{FUNDING}

This research was supported by an operating grant from the Canadian Institutes of Health Research. The Centre for Research on Brain, Language and Music is funded by the government of Quebec via the Fonds de Recherche Nature et Technologies and Société et Culture. MT was supported by scholarships from the Natural Sciences and Engineering Research Council of Canada, the Centre for Research on Brain, Language and Music, and the Fonds de Recherche Nature et Technologies. GG was supported by a scholarship from Conselho Nacional de Desenvolvimento Científico e Tecnológico (CNPq-Brazil).

\section{SUPPLEMENTARY MATERIAL}

The Supplementary Material for this article can be found online at: https://www.frontiersin.org/articles/10.3389/fnsys.2019.000 55/full\#supplementary-material.

thalamic adenosine signaling. Science 356, 1352-1356. doi: 10.1126/science. aaf 4612

Braff, D. L., and Light, G. A. (2004). Preattentional and attentional cognitive deficits as targets for treating schizophrenia. Psychopharmacology 174, 75-85. doi: 10.1007/s00213-004-1848-0

Brozoski, T. J., and Bauer, C. A. (2016). Animal models of tinnitus. Hear. Res. 338, 88-97. doi: 10.1016/j.heares.2015.10.011

Carlson, S., and Willott, J. F. (1996). The behavioral salience of tones as indicated by prepulse inhibition of the startle response: relationship to hearing loss and central neural plasticity in C57BL/6J mice. Hear. Res. 99, 168-175. doi: 10.1016/s0378-5955(96)00098-6

Chen, G., Lee, C., Sandridge, S. A., Butler, H. M., Manzoor, N. F., and Kaltenbach, J. A. (2013). Behavioral evidence for possible simultaneous induction of hyperacusis and tinnitus following intense sound exposure. 
J. Assoc. Res. Otolaryngol. 14, 413-424. doi: 10.1007/s10162-0130375-2

Chung, S., Jeong, J. H., Ko, S., Yu, X., Kim, Y. H., Isaac, J. T. R., et al. (2017). Peripheral sensory deprivation restores critical-period-like plasticity to adult somatosensory thalamocortical inputs. Cell Rep. 19, 2707-2717. doi: 10.1016/j. celrep.2017.06.018

Costigan, M., Scholz, J., and Woolf, C. J. (2009). Neuropathic pain: a maladaptive response of the nervous system to damage. Annu. Rev. Neurosci. 32, 1-32. doi: 10.1146/annurev.neuro.051508.135531

Cramer, S. C., Sur, M., Dobkin, B. H., O’Brien, C., Sanger, T. D., Trojanowski, J. Q., et al. (2011). Harnessing neuroplasticity for clinical applications. Brain 134, 1591-1609. doi: 10.1093/brain/awr039

De Ridder, D., Elgoyhen, A. B., Romo, R., and Langguth, B. (2011). Phantom percepts: tinnitus and pain as persisting aversive memory networks. Proc. Natl. Acad. Sci. U S A 108, 8075-8080. doi: 10.1073/pnas.1018466108

de Villers-Sidani, É., and Merzenich, M. M. (2011). "Lifelong plasticity in the rat auditory cortex: basic mechanisms and role of sensory experience," Progress in Brain Research (Vol. 191), eds A. M. Green, C. E. Chapman, J. F. Kalaska, and F. Lepore (Amsterdam: Elsevier), 119-131.

de Villers-Sidani, E., Simpson, K. L., Lu, Y.-F., Lin, R. C. S., and Merzenich, M. M. (2008). Manipulating critical period closure across different sectors of the primary auditory cortex. Nat. Neurosci. 11, 957-965. doi: 10.1038/nn.2144

Eggermont, J. J. (1992). Neural interaction in cat primary auditory cortex. Dependence on recording depth, electrode separation, and age. J. Neurophysiol. 68, 1216-1228. doi: 10.1152/jn.1992.68.4.1216

Eggermont, J. J. (2007). Correlated neural activity as the driving force for functional changes in auditory cortex. Hear. Res. 229, 69-80. doi: 10.1016/j. heares.2007.01.008

Eggermont, J. J. (2013). On the similarities and differences of non-traumatic sound exposure during the critical period and in adulthood. Front. Syst. Neurosci. 7:12. doi: $10.3389 /$ fnsys.2013.00012

Eggermont, J. J. (2017a). "Animal models of stress and tinnitus," in Tinnitus and Stress: An Interdisciplinary Companion for Healthcare Professionals, eds A. Szczepek and B. Mazurek (Cham: Springer International Publishing), 77-94.

Eggermont, J. J. (2017b). Effects of long-term non-traumatic noise exposure on the adult central auditory system. Hearing problems without hearing loss. Hear. Res. 352, 12-22. doi: 10.1016/j.heares.2016.10.015

Eggermont, J. J., and Roberts, L. E. (2004). The neuroscience of tinnitus. Trends Neurosci. 27, 676-682. doi: 10.1016/j.tins.2004.08.010

Eggermont, J. J., and Smith, G. M. (1996). Neural connectivity only accounts for a small part of neural correlation in auditory cortex. Exp. Brain Res. 110, 379-391. doi: $10.1007 /$ bf 00229138

Elgoyhen, A. B., Langguth, B., De Ridder, D., and Vanneste, S. (2015). Tinnitus: perspectives from human neuroimaging. Nat. Rev. Neurosci. 16, 632-642. doi: $10.1038 / \mathrm{nrn} 4003$

Engineer, N. D., Riley, J. R., Seale, J. D., Vrana, W. A., Shetake, J. A., Sudanagunta, S. P., et al. (2011). Reversing pathological neural activity using targeted plasticity. Nature 470, 101-104. doi: 10.1038/nature09656

Farlow, M. R., and Cummings, J. L. (2007). Effective Pharmacologic Management of Alzheimer's Disease. Am. J. Med. 120, 388-397. doi: 10.1016/j.amjmed.2006.08.036

Flor, H., Denke, C., Schaefer, M., and Grüsser, S. (2001). Effect of sensory discrimination training on cortical reorganisation and phantom limb pain. Lancet 357, 1763-1764. doi: 10.1016/s0140-6736(00)04890-x

Froemke, R. C., Carcea, I., Barker, A. J., Yuan, K., Seybold, B. A., Martins, A. R. O., et al. (2013). Long-term modification of cortical synapses improves sensory perception. Nat. Neurosci. 16, 79-88. doi: 10.1038/nn.3274

Gervain, J., Vines, B. W., Chen, L. M., Seo, R. J., Hensch, T. K., Werker, J. F., et al. (2013). Valproate reopens critical-period learning of absolute pitch. Front. Syst. Neurosci. 7:102. doi: 10.3389/fnsys.2013.00102

Geyer, M. A., and Swerdlow, N. R. (1998). Measurement of startle response, prepulse inhibition, and habituation. Curr. Protoc. Neurosci. 3, 8.7.1-8.7.15. doi: 10.1002/0471142301.ns0807s03

Gourévitch, B., Edeline, J.-M., Occelli, F., and Eggermont, J. J. (2014). Is the din really harmless? Long-term effects of non-traumatic noise on the adult auditory system. Nat. Rev. Neurosci. 15, 483-491. doi: 10.1038/nrn3744

Gu, J. W., Halpin, C. F., Nam, E.-C., Levine, R. A., and Melcher, J. R. (2010). Tinnitus, diminished sound-level tolerance, and elevated auditory activity in humans with clinically normal hearing sensitivity. J. Neurophysiol. 104, 3361-3370. doi: 10.1152/jn.00226.2010

Guercio, G. D., Bevictori, L., Vargas-Lopes, C., Madeira, C., Oliveira, A., Carvalho, V. F., et al. (2014). D-serine prevents cognitive deficits induced by acute stress. Neuropharmacology 86, 1-8. doi: 10.1016/j.neuropharm.2014. 06.021

Guercio, G. D., Thomas, M. E., Cisneros-Franco, J. M., Voss, P., Panizzutti, R., and de Villers-Sidani, E. (2019). Improving cognitive training for schizophrenia using neuroplasticity enhancers: lessons from decades of basic and clinical research. Schizophr. Res. 207, 80-92. doi: 10.1016/j.schres.2018. 04.028

Guest, H., Munro, K. J., Prendergast, G., Howe, S., and Plack, C. J. (2017). Tinnitus with a normal audiogram: relation to noise exposure but no evidence for cochlear synaptopathy. Hear. Res. 344, 265-274. doi: 10.1016/j.heares.2016. 12.002

Han, Y. K., Köver, H., Insanally, M. N., Semerdjian, J. H., and Bao, S. (2007). Early experience impairs perceptual discrimination. Nat. Neurosci. 10, 1191-1197. doi: $10.1038 / \mathrm{nn} 1941$

Harauzov, A., Spolidoro, M., DiCristo, G., De Pasquale, R., Cancedda, L., Pizzorusso, T., et al. (2010). Reducing intracortical inhibition in the adult visual cortex promotes ocular dominance plasticity. J. Neurosci. 30, 361-371. doi: 10.1523/JNEUROSCI.2233-09.2010

He, H.-Y., Hodos, W., and Quinlan, E. M. (2006). Visual deprivation reactivates rapid ocular dominance plasticity in adult visual cortex. J. Neurosci. 26, 2951-2955. doi: 10.1523/JNEUROSCI.5554-05.2006

Hickox, A. E., and Liberman, M. C. (2014). Is noise-induced cochlear neuropathy key to the generation of hyperacusis or tinnitus? J. Neurophysiol. 111, 552-564 doi: $10.1152 /$ jn. 00184.2013

Hofer, S. B., Mrsic-Flogel, T. D., Bonhoeffer, T., and Hübener, M. (2006). Lifelong learning: ocular dominance plasticity in mouse visual cortex. Curr. Opin. Neurobiol. 16, 451-459. doi: 10.1016/j.conb.2006.06.007

Holgers, K., and Pettersson, B. (2005). Noise exposure and subjective hearing symptoms among school children in Sweden. Noise Health 7, 27-37. doi: 10.4103/1463-1741.31635

Kalappa, B. I., Brozoski, T. J., Turner, J. G., and Caspary, D. M. (2014). Single unit hyperactivity and bursting in the auditory thalamus of awake rats directly correlates with behavioural evidence of tinnitus. J. Physiol. 592, 5065-5078. doi: 10.1113/jphysiol.2014.278572

Kaltenbach, J. A. (2011). Tinnitus: models and mechanisms. Hear. Res. 276, 52-60. doi: 10.1016/j.heares.2010.12.003

Kamal, B., Holman, C., and de Villers-Sidani, E. (2013). Shaping the aging brain: role of auditory input patterns in the emergence of auditory cortical impairments. Front. Syst. Neurosci. 7:52. doi: 10.3389/fnsys.2013. 00052

Keuroghlian, A. S., and Knudsen, E. I. (2007). Adaptive auditory plasticity in developing and adult animals. Prog. Neurobiol. 82, 109-121. doi: 10.1016/j. pneurobio.2007.03.005

Kilgard, M. P., Vazquez, J. L., Engineer, N. D., and Pandya, P. K. (2007). Experience dependent plasticity alters cortical synchronization. Hear. Res. 229, 171-179. doi: 10.1016/j.heares.2007.01.005

Kuner, R., and Flor, H. (2016). Structural plasticity and reorganisation in chronic pain. Nat. Rev. Neurosci. 18, 20-30. doi: 10.1038/nrn.2016.162

Lakens, D. (2013). Calculating and reporting effect sizes to facilitate cumulative science: a practical primer for t-tests and ANOVAs. Front. Psychol. 4:863. doi: 10.3389/fpsyg.2013.00863

Langers, D. R. M., de Kleine, E., and van Dijk, P. (2012). Tinnitus does not require macroscopic tonotopic map reorganization. Front. Syst. Neurosci. 6:2 doi: 10.3389 /fnsys.2012.00002

Lau, C., Zhang, J. W., McPherson, B., Pienkowski, M., and Wu, E. X. (2015). Long-term, passive exposure to non-traumatic acoustic noise induces neural adaptation in the adult rat medial geniculate body and auditory cortex. Neuroimage 107, 1-9. doi: 10.1016/j.neuroimage.2014.11.048

Longenecker, R. J., and Galazyuk, A. V. (2012). Methodological optimization of tinnitus assessment using prepulse inhibition of the acoustic startle reflex. Brain Res. 1485, 54-62. doi: 10.1016/j.brainres.2012.02.067

Longenecker, R. J., and Galazyuk, A. V. (2016). Variable effects of acoustic trauma on behavioral and neural correlates of tinnitus in individual animals. Front. Behav. Neurosci. 10:207. doi: 10.3389/fnbeh.2016.00207 
Massoud, F., and Léger, G. C. (2011). Pharmacological Treatment of Alzheimer Disease. Can. J. Psychiatry. 56, 579-588. doi: 10.1177/070674371105601003

Merzenich, M. M., Van Vleet, T. M., and Nahum, M. (2014). Brain plasticity-based therapeutics. Front. Hum. Neurosci. 8:385. doi: 10.3389/fnhum.2014.00385

Moore, D. R., Zobay, O., Mackinnon, R. C., Whitmer, W. M., and Akeroyd, M. A. (2017). Lifetime leisure music exposure associated with increased frequency of tinnitus. Hear. Res. 347, 18-27. doi: 10.1016/j.heares.2016.10.030

Noreña, A. J., and Eggermont, J. J. (2003). Changes in spontaneous neural activity immediately after an acoustic trauma: implications for neural correlates of tinnitus. Hear. Res. 183, 137-153. doi: 10.1016/s0378-5955(03)00225-9

Noreña, A. J., and Eggermont, J. J. (2006). Enriched acoustic environment after noise trauma abolishes neural signs of tinnitus. Neuroreport 17, 559-563. doi: 10.1097/00001756-200604240-00001

Nowak, L. G., and Bullier, J. (2005). "Cross correlograms for neuronal spike trains: different types of temporal correlation in neocortex, their origin and significance," in Time and the Brain, ed. R. Miller (Amsterdam: Harwood Academic Publishers), 53-96.

O'Reilly, K. C., Perica, M. I., and Fenton, A. A. (2019). Synaptic plasticity/dysplasticity, process memory and item memory in rodent models of mental dysfunction. Schizophr. Res. 207, 22-36. doi: 10.1016/j.schres.2018. 08.025

Pienkowski, M. (2018). Prolonged exposure of CBA/Ca mice to moderately loud noise can cause cochlear synaptopathy but not tinnitus or hyperacusis as assessed with the acoustic startle reflex. Trends Hear. 22:2331216518758109. doi: $10.1177 / 2331216518758109$

Pienkowski, M., and Eggermont, J. J. (2009). Long-term, partially-reversible reorganization of frequency tuning in mature cat primary auditory cortex can be induced by passive exposure to moderate-level sounds. Hear. Res. 257, 24-40. doi: 10.1016/j.heares.2009.07.011

Pienkowski, M., and Eggermont, J. J. (2010). Intermittent exposure with moderatelevel sound impairs central auditory function of mature animals without concomitant hearing loss. Hear. Res. 261, 30-35. doi: 10.1016/j.heares.2009. 12.025

Pienkowski, M., and Eggermont, J. J. (2011). Cortical tonotopic map plasticity and behavior. Neurosci. Biobehav. Rev. 35, 2117-2128. doi: 10.1016/j.neubiorev. 2011.02.002

Pienkowski, M., and Eggermont, J. J. (2012). Reversible long-term changes in auditory processing in mature auditory cortex in the absence of hearing loss induced by passive, moderate-level sound exposure. Ear Hear. 33, 305-314. doi: 10.1097/aud.0b013e318241e880

Pienkowski, M., Munguia, R., and Eggermont, J. J. (2011). Passive exposure of adult cats to bandlimited tone pip ensembles or noise leads to long-term response suppression in auditory cortex. Hear. Res. 277, 117-126. doi: 10.1016/j.heares.2011.02.002

Pizzorusso, T., Medini, P., Berardi, N., Chierzi, S., Fawcett, J. W., and Maffei, L. (2002). Reactivation of ocular dominance plasticity in the adult visual cortex. Science 298, 1248-1251. doi: 10.1126/science. 1072699

Polley, D. B., Read, H. L., Storace, D. A., and Merzenich, M. M. (2007). Multiparametric auditory receptive field organization across five cortical fields in the albino rat. J. Neurophysiol. 97, 3621-3638. doi: 10.1152/jn. 01298.2006

Profant, O., Burianová, J., and Syka, J. (2013). The response properties of neurons in different fields of the auditory cortex in the rat. Hear. Res. 296, 51-59. doi: 10.1016/j.heares.2012.11.021

Reed, A., Riley, J., Carraway, R., Carrasco, A., Perez, C., Jakkamsetti, V., et al. (2011). Cortical map plasticity improves learning but is not necessary for improved performance. Neuron 70, 121-131. doi: 10.1016/j.neuron.2011. 02.038

Reed, J. L., and Kaas, J. H. (2010). Statistical analysis of large-scale neuronal recording data. Neural Netw. 23, 673-684. doi: 10.1016/j.neunet.2010.04.005

Reinhard, S. M., Abundez-Toledo, M., Espinoza, K., and Razak, K. A. (2019). Effects of developmental noise exposure on inhibitory cell densities and perineuronal nets in A1 and AAF of mice. Hear. Res. 381:107781. doi: 10.1016/j. heares.2019.107781
Roberts, L. E., Eggermont, J. J., Caspary, D. M., Shore, S. E., Melcher, J. R., and Kaltenbach, J. A. (2010). Ringing ears: the neuroscience of tinnitus. J. Neurosci. 30, 14972-14979. doi: 10.1523/JNEUROSCI.4028-10. 2010

Sun, W., Deng, A., Jayaram, A., and Gibson, B. (2012). Noise exposure enhances auditory cortex responses related to hyperacusis behavior. Brain Res. 1485, 108-116. doi: 10.1016/j.brainres.2012.02.008

Swerdlow, N. R., Braff, D. L., and Geyer, M. A. (2000). Animal models of deficient sensorimotor gating: what we know, what we think we know, and what we hope to know soon. Behav. Pharmacol. 11, 185-204. doi: 10.1097/00008877200006000-00002

Swerdlow, N. R., and Light, G. A. (2018). Sensorimotor gating deficits in schizophrenia: advancing our understanding of the phenotype, its neural circuitry and genetic substrates. Schizophr. Res. 198, 1-5. doi: 10.1016/j.schres. 2018.02.042

Syka, J., and Rybalko, N. (2000). Threshold shifts and enhancement of cortical evoked responses after noise exposure in rats. Hear. Res. 139, 59-68. doi: 10.1016/s0378-5955(99)00175-6

Tabot, G. A., Kim, S. S., Winberry, J. E., and Bensmaia, S. J. (2015). Restoring tactile and proprioceptive sensation through a brain interface. Neurobiol. Dis. 83, 191-198. doi: 10.1016/j.nbd.2014.08.029

Takahashi, K., Hishida, R., Kubota, Y., Kudoh, M., Takahashi, S., and Shibuki, K. (2006). Transcranial fluorescence imaging of auditory cortical plasticity regulated by acoustic environments in mice. Eur. J. Neurosci. 23, 1365-1376. doi: $10.1111 / j .1460-9568.2006 .04662 . x$

Takahashi, H., Yokota, R., Funamizu, A., Kose, H., and Kanzaki, R. (2011). Learning-stage-dependent, field-specific, map plasticity in the rat auditory cortex during appetitive operant conditioning. Neuroscience 199, 243-258. doi: 10.1016/j.neuroscience.2011.09.046

Thomas, M. E., Friedman, N. H., Cisneros-Franco, J. M., Ouellet, L., and de Villers-Sidani, É. (2018). The prolonged masking of temporal acoustic inputs with noise drives plasticity in the adult rat auditory cortex. Cereb. Cortex 29, 1032-1046. doi: 10.1093/cercor/ bhy009

Turner, J. G., and Larsen, D. (2016). Effects of noise exposure on development of tinnitus and hyperacusis: prevalence rates 12 months after exposure in middle-aged rats. Hear. Res. 334, 30-36. doi: 10.1016/j.heares.2015. 11.004

Turner, J. G., and Parrish, J. (2008). Gap detection methods for assessing salicylateinduced tinnitus and hyperacusis in rats. Am. J. Audiol. 17, S185-S192. doi: 10.1044/1059-0889(2008/08-0006)

Wang, H., Brozoski, T. J., and Caspary, D. M. (2011). Inhibitory neurotransmission in animal models of tinnitus: maladaptive plasticity. Hear. Res. 279, 111-117. doi: 10.1016/j.heares.2011.04.004

Zheng, W. (2012). Auditory map reorganization and pitch discrimination in adult rats chronically exposed to low-level ambient noise. Front. Syst. Neurosci. 6:65. doi: 10.3389/fnsys.2012.00065

Zhou, X., and Merzenich, M. M. (2012). Environmental noise exposure degrades normal listening processes. Nat. Commun. 3:843. doi: 10.1038/ncomms1849

Zhou, X., Panizzutti, R., de Villers-Sidani, E., Madeira, C., and Merzenich, M. M. (2011). Natural restoration of critical period plasticity in the juvenile and adult primary auditory cortex. J. Neurosci. 31, 5625-5634. doi: 10.1523/JNEUROSCI. 6470-10.2011

Conflict of Interest: The authors declare that the research was conducted in the absence of any commercial or financial relationships that could be construed as a potential conflict of interest.

Copyright (c) 2019 Thomas, Guercio, Drudik and de Villers-Sidani. This is an open-access article distributed under the terms of the Creative Commons Attribution License (CC BY). The use, distribution or reproduction in other forums is permitted, provided the original author(s) and the copyright owner(s) are credited and that the original publication in this journal is cited, in accordance with accepted academic practice. No use, distribution or reproduction is permitted which does not comply with these terms. 